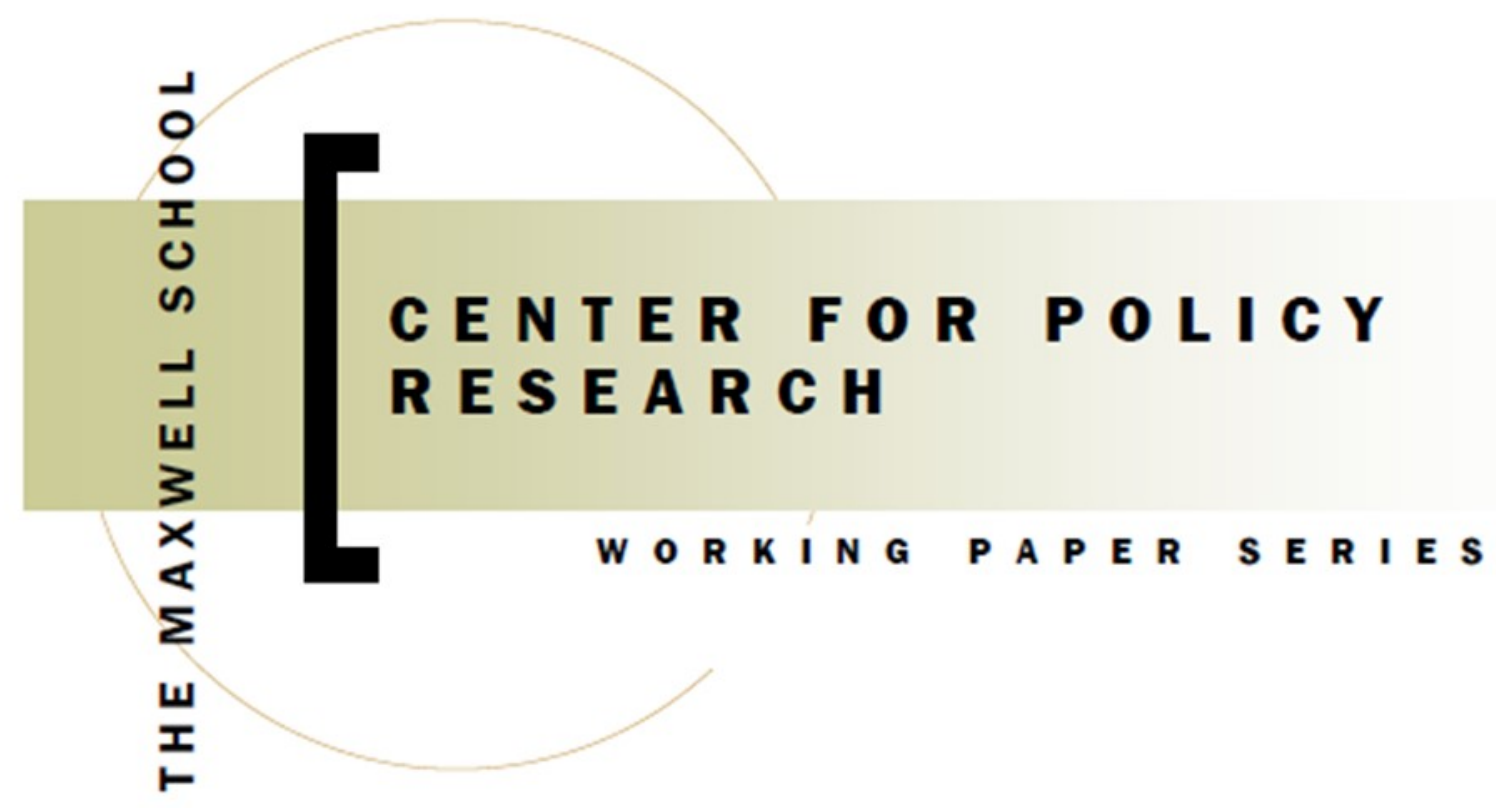

\title{
Test of Hypotheses in a Time Trend Panel Data Model with Serially Corre- lated Error Component Disturbances
}

Badi H. Baltagi, Chihwa Kao, and Long Liu

\section{IS N N $1525-3066$}

426 Eggers Hall

Syracuse University

Syracuse, NY 13244-1020

(315) 443-3114 / email: ctrpol@syr.edu 


\section{CENTER FOR POLICY RESEARCH -Summer 2014}

\section{Leonard M. Lopoo, Director \\ Associate Professor of Public Administration and International Affairs (PAIA)}

\section{Associate Directors}

Margaret Austin

Associate Director

Budget and Administration

John Yinger

Trustee Professor of Economics and PAIA

Associate Director, Metropolitan Studies Program

\section{SENIOR RESEARCH ASSOCIATES}

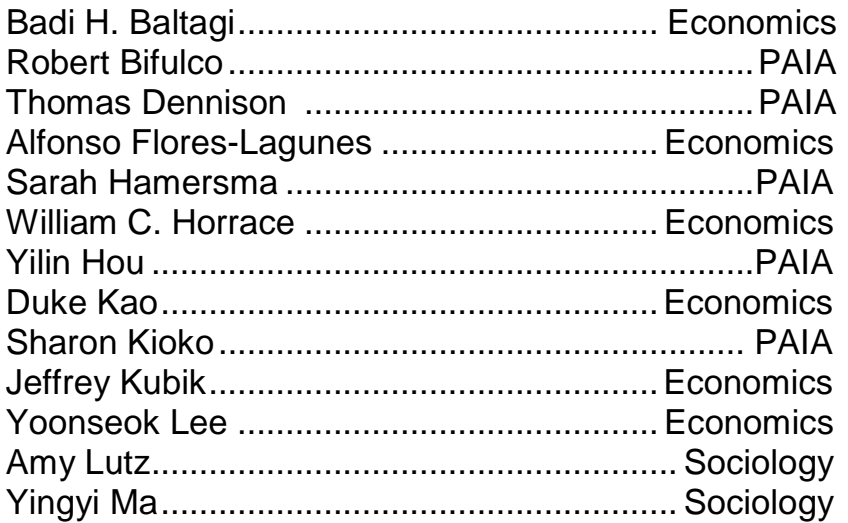

Jerry Miner

Cynthia Morrow

Jan Ondrich.

John Palmer

David Popp

Stuart Rosenthal

Ross Rubenstein

Rebecca Schewe

Amy Ellen Schwartz

Perry Singleton.

Abbey Steele.

Michael Wasylenko....

Peter Wilcoxen.
Economics PAIA

Economics

PAIA

PAIA

Economics PAIA

Sociology PAIA/Economics Economics PAIA Economics PAIA

\section{GRADUATE ASSOCIATES}

Emily Cardon

Hannah Dalager

Maidel De La Cruz.

Carlos Diaz.

Vantiel Elizabeth Duncan

Alex Falevich ....

Lincoln Groves

Ruby Jennings

Yusun Kim

Bridget Lenkiewicz

Michelle Lofton

Roberto Martinez
PAIA Qing Miao

PAIA Nuno Abreu Faro E Mota

PAIA Judson Murchie

Economics

PAIA

Economics

PAIA

PAIA

PAIA

PAIA

PAIA

PAIA
Sun Jung Oh

Brian Ohl.

Laura Rodriquez-Ortiz

Timothy Smilnak

Kelly Stevens....

Rebecca Wang

Pengju Zhang

Xirui Zhang
PAIA

Economics PAIA

Social Science PAIA

PAIA

PAIA

PAIA

Sociology

Economics

Economics

\section{STAFF}

Kelly Bogart.

Karen Cimilluca.

Kathleen Nasto
..Administrative Specialist Office Coordinator Administrative Assistant
Candi Patterson. Computer Consultant Mary Santy.... Katrina Wingle
Administrative Assistant Administrative Assistant 


\section{Abstract}

This paper studies test of hypotheses for the slope parameter in a linear time trend panel data model with serially correlated error component disturbances. We propose a test statistic that uses a bias corrected estimator of the serial correlation parameter. The proposed test statistic which is based on the corresponding fixed effects feasible generalized least squares (FE-FGLS) estimator of the slope parameter has the standard normal limiting distribution which is valid whether the remainder error is $\mathrm{I}(0)$ or $\mathrm{I}(1)$. This performs well in Monte Carlo experiments and is recommended.

JEL No. C23, C33

Keywords: Panel Data, Generalized Least Squares, Time Trend Model, Fixed Effects, First Difference, and Nonstationarity.

We dedicate this paper in honor of Peter C.B. Phillips many contributions to econometrics and in particular non-stationary time series analysis and panel data. We would like to thank an anonymous referee and the editor Tom Fomby for their helpful suggestions.

Address correspondence to: Badi H. Baltagi, Department of Economics and Center for Policy Research, 426 Eggers Hall, Syracuse University, Syracuse, NY 13244-1020; tel: 315-443-1630; fax: 315-443-1081; e-mail: bbaltagi@maxwell.syr.edu.

Chihwa Kao: Department of Economics, Center for Policy Research, 426 Eggers Hall, Syracuse University, Syracuse, NY 13244-1020; tel: 315-443-3233; fax: 315-443-1081; e-mail: cdkao@maxwell.syr.edu.

Long Liu: Department of Economics, College of Business, University of Texas at San Antonio, One UTSA Circle, TX 78249-0633; tel: 210-458-6169; fax: 210-458-5837; e-mail: long.liu@utsa.edu. 


\title{
Test of Hypotheses in a Time Trend Panel Data Model with Serially Correlated Error Component Disturbances*
}

\author{
Badi H. Baltagi ${ }^{\dagger}$ Chihwa Kaoł Long Liu ${ }^{\S}$
}

This version: June 22, 2014

\begin{abstract}
This paper studies test of hypotheses for the slope parameter in a linear time trend panel data model with serially correlated error component disturbances. We propose a test statistic that uses a bias corrected estimator of the serial correlation parameter. The proposed test statistic which is based on the corresponding fixed effects feasible generalized least squares (FE-FGLS) estimator of the slope parameter has the standard normal limiting distribution which is valid whether the remainder error is $I(0)$ or $I(1)$. This performs well in Monte Carlo experiments and is recommended.

Keywords: Panel Data, Generalized Least Squares, Time Trend Model, Fixed Effects, First Difference, Nonstationarity.
\end{abstract}

JEL Classification: C23, C33.

\section{Introduction}

Panel data regression models with large cross-sectional and time-series dimensions have attracted much attention in recent years, e.g., see the surveys by Baltagi and Kao (2000), Phillips and Moon (2000), Choi (2006) and Breitung and Pesaran (2008) to mention a few. ${ }^{1}$ Phillips and Moon (1999) provide joint asymptotic analysis of pooled estimators in panel regressions with non-stationary regressors when the underlying regression disturbances follow stationary processes. Under the additional condition, $n / T \rightarrow 0$, they show that sequential asymptotic results for their pooled

\footnotetext{
${ }^{*}$ We dedicate this paper in honour of Peter C.B. Phillips's many contributions to econometrics and in particular non-stationary time series analysis and panel data. We would like to thank an anonymous referee and the editor Tom Fomby for their helpful suggestions.

${ }^{\dagger}$ Address correspondence to: Badi H. Baltagi, Department of Economics and Center for Policy Research, 426 Eggers Hall, Syracuse University, Syracuse, NY 13244-1020; tel: 315-443-1630; fax: 315-443-1081; e-mail: bbaltagi@maxwell.syr.edu.

${ }^{\ddagger}$ Chihwa Kao: Department of Economics, Center for Policy Research, 426 Eggers Hall, Syracuse University, Syracuse, NY 13244-1020; tel: 315-443-3233; fax: 315-443-1081; e-mail: cdkao@maxwell.syr.edu.

${ }^{\S}$ Long Liu: Department of Economics, College of Business, University of Texas at San Antonio, One UTSA Circle, TX 78249-0633; tel: 210-458-6169; fax: 210-458-5837; e-mail: long.liu@utsa.edu.

${ }^{1}$ See also chapter 12 of Baltagi (2008) for a textbook treatment of this subject.
} 
estimators would be equivalent to the joint ones. Kao and Emerson (2004) and Baltagi, Kao and Liu (2008) show that the asymptotics of the standard panel data estimators, like the fixed effects (FE), first-difference (FD) and generalized least squares (GLS) estimators of the slope coefficient depend crucially upon whether the error term is $I(0)$ or $I(1)$. For example, when the error term is $I(0)$, the FE and GLS estimators are asymptotically equivalent. However, when the error term is $I(1)$, this asymptotic equivalence breaks down and the GLS estimator is more efficient than the FE estimator. This paper considers fixed effects GLS (FE-GLS) based test statistics to test hypotheses regarding the slope parameter of a panel data time trend model where apriori knowledge as to whether the errors are $I(0)$ or $I(1)$ is not available. ${ }^{2}$ We discuss the asymptotic properties of estimators of the autoregressive parameter and the corresponding fixed effects feasible GLS (FEFGLS) estimators of the slope parameter. The main contribution of this paper is to introduce a FE-FGLS based test statistic which is robust when the error term is either $I(0)$ or $I(1)$.

The paper is organized as follows: Section 2 presents the panel data time trend model with an $\mathrm{AR}(1)$ error term. In Section 3, we discuss the FE-GLS estimator for this model and propose a test statistic that uses a bias corrected estimator of the serial correlation parameter and the corresponding FE-FGLS estimator of the regression parameter. Monte Carlo simulations are given in Section 4, while Section 5 provides the concluding remarks. All the proofs are given in the Appendix. A few words on notation. All limits are taken sequentially as $T \rightarrow \infty$ and $n \rightarrow \infty$ unless otherwise specified. We use $(n, T) \rightarrow \infty$ to denote the sequential limit. Convergence in

probability and distribution are denoted by $\stackrel{p}{\rightarrow}$ and $\stackrel{d}{\rightarrow}$, respectively. The limiting distribution of double indexed integrated processes has been extensively studied by Phillips and Moon (1999, 2000).

\section{The Model}

Consider the following panel data time trend model:

$$
y_{i t}=\delta+\beta t+u_{i t}, \quad i=1, \ldots, n, \quad t=1, \ldots, T,
$$

\footnotetext{
${ }^{2}$ The results in this paper make use of the asymptotic results for a panel data time trend regression model studied by Kao and Emerson (2004).
} 
where $u_{i t}=\mu_{i}+\nu_{i t}$, and $\delta$ and $\beta$ are scalars. We assume that the individual effects $\mu_{i}$ are random with $\mu_{i} \sim i i d\left(0, \sigma_{\mu}^{2}\right)$ and $\nu_{i t}$ following an $\mathrm{AR}(1)$ process which may or may not be stationary

$$
\nu_{i t}=\rho \nu_{i t-1}+e_{i t}
$$

with $|\rho| \leq 1$, where $e_{i t}$ is a white noise process with variance $\sigma_{e}^{2}$. The $\mu_{i}$ 's are independent of the $\nu_{i t}$ 's for all $i$ and $t$. This model has been studied by Baltagi and Krämer (1997) and Kao and Emerson (2004). In fact, Baltagi and Krämer (1997) showed the equivalence of OLS, GLS and FE estimators for the panel data time trend model (1), but without serial correlation. Baltagi and Krämer (1997) also investigated the relative efficiency of the FD estimator with respect to the other estimators of $\beta$ as $T \rightarrow \infty$. Kao and Emerson (2004) extended Baltagi and Krämer to model (1) with serially correlated remainder errors (2). ${ }^{3}$ They showed that the FE estimator is asymptotically equivalent to GLS when the error term is $I(0)$ but that GLS is more efficient than FE when the error term is $I(1)$. Kao and Emerson show that the properties of the standard panel data estimators, like the FE, FD, and GLS estimators of $\beta$ depend crucially upon the value of $\rho .{ }^{4}$ When $\nu_{i t}$ is $I(0)$, i.e., $\rho<1$, the FE and GLS estimators are both $\sqrt{n} T^{3 / 2}$ consistent and asymptotically equivalent. However, when $\nu_{i t}$ is $I(1)$, i.e., $\rho=1$, this asymptotic equivalence breaks down and the GLS estimator is more efficient than the FE estimator. This has serious implications for applied research when $\nu_{i t}$ is serially correlated and it is unknown whether the remainder disturbances are $I(0)$ or $I(1) .^{5}$

In this paper, we are interested in testing

$$
H_{0}: \beta=\beta_{0}
$$

without assuming knowledge of whether $v_{i t}$ is $I(0)$ or $I(1) .{ }^{6}$ Hypothesis testing on the slope of

\footnotetext{
${ }^{3}$ One can extend the simple time trend model in this paper to a polynomial trend model by following similar steps as in section 6 of Emerson and Kao (2000).

${ }^{4}$ Baltagi, Kao and Liu (2008) study the asymptotic properties of OLS, FE, FD and GLS in the random effects error components regression model with an autocorrelated regressor and an autocorrelated remainder error (both of which can be stationary or nonstationary). They show that when the error term is $I(0)$ and the regressor is $I(1)$, the FE estimator is asymptotically equivalent to the GLS estimator and OLS is less efficient than GLS (due to a slower convergence speed). However, when the error term and the regressor are $I(1)$, GLS is more efficient than the FE estimator since GLS is $\sqrt{n T}$ consistent, while FE is $\sqrt{n}$ consistent. This implies that GLS is the preferred estimator under both cases (i.e., regression error is either $I(0)$ or $I(1)$ ).

${ }^{5}$ One referee suggest testing the joint hypothesis: $H_{0}: \beta=0$ and $\rho=0$. Alternatively, testing the joint hypothesis: $H_{0}: \beta=0$ and $\rho=1$. Extending the results of this paper to these joint hypotheses is beyond the scope of this paper and should be subject for future research.

${ }^{6}$ Baltagi, Kao and $\mathrm{Na}$ (2011) also consider hypotheses testing in an $\mathrm{I}(0)$ or $\mathrm{I}(1)$ regressor case. However, the
} 
the trend has been studied in the econometric time series literature, e.g., Canjels and Watson (1997), Vogelsang and Fomby (2002), Bunzel and Vogelsang (2005), Roy, Falk and Fuller (2004), and Perron and Yabu (2009). The focus of this paper is on the corresponding test in panel data. Consider the FE estimator of $\beta$, which is given by

$$
\hat{\beta}_{F E}=\frac{\sum_{i=1}^{n} \sum_{t=1}^{T}(t-\bar{t})\left(y_{i t}-\bar{y}_{i}\right)}{n \sum_{t=1}^{T}(t-\bar{t})^{2}},
$$

where $\bar{t}=\frac{1}{T} \sum_{t=1}^{T} t$ and $\bar{y}_{i}=\frac{1}{T} \sum_{t=1}^{T} y_{i t}{ }^{7}$ If $v_{i t}$ is known to be $I(0)$, the $t$-statistic for the null hypothesis $H_{0}$ can be constructed using the FE estimator as follows:

$$
t_{F E}=\frac{\hat{\beta}_{F E}-\beta_{0}}{\sqrt{\operatorname{Var}\left(\hat{\beta}_{F E}\right)}},
$$

where $\operatorname{Var}\left(\widehat{\beta}_{F E}\right)=\frac{\hat{\sigma}_{v}^{2}}{n \sum_{t=1}^{T}(t-\bar{t})^{2}}$ with $\hat{\sigma}_{v}^{2}=\frac{1}{n(T-1)-1} \sum_{i=1}^{n} \sum_{t=2}^{T} \hat{\nu}_{i t}^{2}$ and $\hat{\nu}_{i t}$ are the within residuals from (1), i.e., $\hat{\nu}_{i t}=\left(y_{i t}-\bar{y}_{i}\right)-\hat{\beta}_{F E}(t-\bar{t})$. The next theorem derives the limiting distribution of $t_{F E}$ when the error term is $I(0)$ or $I(1)$.

Theorem 1 Assume $(n, T) \rightarrow \infty$,

1. If $|\rho|<1$

$$
t_{F E} \stackrel{d}{\rightarrow} N(0,1)
$$

2. If $\rho=1$,

$$
\frac{1}{\sqrt{T}} t_{F E} \stackrel{d}{\rightarrow} N\left(0, \frac{3}{5}\right)
$$

From Theorem 1, we note that $t_{F E}$ in (4) will converge to a standard normal only when $v_{i t}$ is $I(0)$, and $t_{F E}$ will diverge when the error term is $I(1)$. This is not surprising since $\hat{\sigma}_{v}^{2}=O_{p}(T)$, i.e., $\sigma_{v}^{2}$ is not identified when $v_{i t}$ is $I(1)$. If $v_{i t}$ is known to be $I(1)$, then the optimal test for testing the null hypothesis $H_{0}$ is based on the t-statistic using the FD estimator, $\hat{\beta}_{F D}$, which is given by

$$
\hat{\beta}_{F D}=\frac{1}{n T} \sum_{i=1}^{n} \sum_{t=1}^{T} \Delta y_{i t},
$$

results in this paper for a time trend panel data model with serially correlated error component disturbances are different. For example, we show that the GLS based t-statistics with individual fixed effects have a different limiting distribution compared to that without the fixed effects.

${ }^{7}$ Note that the FE and GLS estimators are asymptotically equivalent for this case, see Kao and Emerson (2004). 
where $\Delta y_{i t}=y_{i t}-y_{i, t-1}$. The corresponding $t$-statistic is given by:

$$
t_{F D}=\frac{\hat{\beta}_{F D}-\beta_{0}}{\sqrt{\operatorname{Var}\left(\hat{\beta}_{F D}\right)}},
$$

where $\operatorname{Var}\left(\hat{\beta}_{F D}\right)=\frac{\hat{\sigma}_{e}^{2}}{n T}$ with $\hat{\sigma}_{e}^{2}=\frac{1}{n(T-1)-1} \sum_{i=1}^{n} \sum_{t=2}^{T}\left(\Delta y_{i t}-\hat{\beta}_{F D}\right)^{2}$. The next theorem derives the limiting distribdtion of $t_{F D}$ when the error term is $I(0)$ or $I(1)$.

Theorem 2 Assume $(n, T) \rightarrow \infty$,

1. If $|\rho|<1$,

$$
\sqrt{T} t_{F D} \stackrel{d}{\rightarrow} N\left(0, \frac{1}{1-\rho}\right)
$$

2. If $\rho=1$,

$$
t_{F D} \stackrel{d}{\rightarrow} N(0,1)
$$

The results of Theorem 2 show that $t_{F D} \stackrel{d}{\rightarrow} N(0,1)$ when $v_{i t}$ is in fact $I(1)$, under the null. On the other hand, $t_{F D} \stackrel{d}{\rightarrow} 0$ if $v_{i t}$ is $I(0)$, under the null. In view of this and given that the order of integration of $v_{i t}$ is not known in practice, it is natural to consider alternative robust test procedures.

\section{The FE-FGLS Estimator}

Rewrite equation (1) in matrix form as

$$
y=\delta \iota_{n T}+\beta x+u
$$

with $u=Z_{\mu} \mu+\nu$, where $u^{\prime}=\left(u_{11}, \ldots, u_{1 T}, u_{21}, \ldots, u_{2 T}, \ldots, u_{n 1}, \ldots, u_{n T}\right)$ with the observations stacked such that the slower index is over individuals and the faster index is over time. $\mu$ is an $n \times 1$ vector with typical element $\mu_{i}, \boldsymbol{\nu}$ is an $n T \times 1$ vector with typical element $\nu_{i t}$, and $Z_{\mu}=I_{n} \quad \iota_{T}$, where $I_{n}$ is an identity matrix of dimension $n, \iota_{T}$ is a vector of ones of dimension $T$, and denotes the Kronecker product. $y$ is an $n T \times 1$ vector with typical element $y_{i t}, x=\iota_{n} \quad x_{i}$, where $\iota_{n}$ is a vector of ones of dimension $n$ and $\mathbf{x}_{i}$ is a $T \times 1$ vector indicating a time trend with elements $(1,2, \ldots, T) \cdot \boldsymbol{\iota}_{n T}$ is a vector of ones of dimension $n T$. As shown in Baltagi and Li (1991), one can write the variance-covariance matrix as

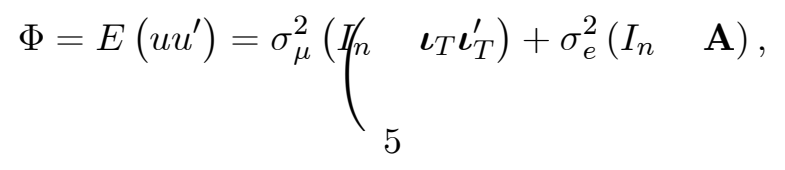


where $\mathbf{A}$ is the variance-covariance matrix of $v_{i t}$, i.e.,

when $|\rho|<1$ and

$$
\mathbf{A}=\frac{1}{1-\rho^{2}}\left[\begin{array}{ccccc}
1 & \rho & \rho^{2} & \cdots & \rho^{T-1} \\
\rho & 1 & \rho & \cdots & \rho^{T-2} \\
\rho^{2} & \rho & 1 & \cdots & \rho^{T-3} \\
\vdots & \vdots & \vdots & \ddots & \vdots \\
\oint^{T-1} & \rho^{T-2} & \rho^{T-3} & \cdots & 1
\end{array}\right]
$$

when $\rho=1$. When $|\rho|<1$, one can easily verify that $\mathbf{A}^{-1}=\mathbf{C}^{\prime} \mathbf{C}$, where

$$
\mathbf{C}=\left[\begin{array}{cccccc}
\sqrt{1-\rho^{2}} & 0 & 0 & \cdots & 0 & 0 \\
-\rho & 1 & 0 & \cdots & 0 & 0 \\
0 & -\rho & 1 & \cdots & 0 & 0 \\
\vdots & \vdots & \vdots & \ddots & \vdots & \vdots \\
0 & 0 & 0 & -\rho & 1 & 0 \\
0 & 0 & 0 & 0 & -\rho & 1
\end{array}\right]
$$

is the Prais-Winsten transformation matrix as in Baltagi and Li (1991). As suggested in Baltagi and Li (1991), one can apply the Prais-Winsten transformation matrix $\mathbf{C}$ to transform the remainder $\mathrm{AR}(1)$ disturbances into serially uncorrelated classical errors:

$$
y^{*}=\delta \iota_{n T}^{*}+\beta x^{*}+u^{*}
$$

where $y^{*}=\left(\begin{array}{ll}I_{n} & C\end{array}\right) y, x^{*}=\left(\begin{array}{ll}I_{n} & C\end{array}\right) x=\iota_{n} \quad x_{i}^{*}$ with $x_{i}^{*}=C x_{i}$ and $\iota_{n T}^{*}=\left(\begin{array}{ll}I_{n} & C\end{array}\right) \iota_{n T}=$ $(1-\rho)\left(\begin{array}{ll}\boldsymbol{\iota}_{n} & \boldsymbol{\iota}_{T}^{\alpha}\end{array}\right)$ using the fact that $C \boldsymbol{\iota}_{T}=(1-\rho) \boldsymbol{\iota}_{T}^{\alpha}$, where $\boldsymbol{\iota}_{T}^{\alpha^{\prime}}=\left(\not, \boldsymbol{\iota}_{T-1}^{\prime}\right)$ find $\alpha=\sqrt{(1+\rho) /(1-\rho)}$.
The transformed regression disturbances are in vector form

$$
u^{*}=\left(\begin{array}{ll}
I_{n} & C
\end{array}\right) u=\left(\begin{array}{ll}
I_{n} & C \boldsymbol{\iota}_{T}
\end{array}\right) \mu+\left(\begin{array}{ll}
I_{n} & C
\end{array}\right) v=(1-\rho)\left(\begin{array}{ll}
I_{n} & \boldsymbol{\iota}_{T}^{\alpha}
\end{array}\right) \mu+v^{*},
$$


where $v^{*}=\left(\begin{array}{ll}I_{n} & C\end{array}\right) v$. As shown in Baltagi and Li (1991), the variance-covariance matrix of the transformed disturbance is

and

$$
\Phi^{*}=E\left(u^{*} u^{* \prime}\right)\left(=\sigma_{\mu}^{2}(1-\rho)^{2}\left(\begin{array}{ll}
I_{n} & \iota_{T}^{\alpha} \iota_{T}^{\alpha \prime}
\end{array}\right)\left(\begin{array}{ll}
+\sigma_{\varepsilon}^{2}\left(I_{n}\right. & I_{T}
\end{array}\right)\right.
$$

$$
\sigma_{\varepsilon} \Phi^{*-1 / 2}=\frac{\sigma_{\varepsilon}}{\sigma_{\alpha}}\left(\begin{array}{ll}
I_{n} & \bar{J}_{T}^{\alpha}
\end{array}\right)\left(\begin{array}{ll}
I_{n} & E_{T}^{\alpha}
\end{array}\right)
$$

where $E_{T}^{\alpha}=I_{T}-\bar{J}_{T}^{\alpha}, \bar{J}_{T}^{\alpha}=\iota_{T}^{\alpha} \iota_{T}^{\alpha \prime} / d^{2}, d^{2}=\iota_{T}^{\alpha \prime} \iota_{T}^{\alpha}=\alpha^{2}+T-1, \sigma_{\alpha}^{2}=\sigma_{e}^{2}+\theta \sigma_{\mu}^{2}$ and $\theta=d^{2}(1-\rho)^{2}$. Premultiplying the PW transformed observations in Equation (11) by $\sigma_{\varepsilon} \Phi^{*-1 / 2}$, one gets

$$
\sigma_{\varepsilon} \Phi^{*-1 / 2} y^{*}=\sigma_{\varepsilon} \Phi^{*-1 / 2} \delta \iota_{n T}^{*}+\sigma_{\varepsilon} \Phi^{*-1 / 2} \beta x^{*}+\sigma_{\varepsilon} \Phi^{*-1 / 2} u^{*}
$$

The least squares estimator of the transformed equation yields the GLS estimator $\hat{\beta}_{G L S}$. As shown in Baltagi, Kao and Liu (2008), $\hat{\beta}_{G L S}$ has a faster converging speed than both the FE and FD estimators as $(n, T) \rightarrow \infty$. This is true whether $v_{i t}$ and $x_{i t}$ are $I(1)$ or $I(0)$. Baltagi, Kao and Na (2011) further showed that the t-test statistic for $H_{0}: \beta=\beta_{0}$ based on $\hat{\beta}_{G L S}$ is always $N(0,1)$ as $(n, T) \rightarrow \infty$.

A critical assumption for the GLS estimator is that $E\left(\mu_{i} \mid x_{i t}\right)=0$. It is well known that when there is correlation between the regressors and the individual effects, GLS suffers from omitted variable bias, while FD and FE wipe out this source of endogeneity and remain consistent. In case of serial correlation, Baltagi, Kao and Liu (2008) suggest a FE-GLS estimator that uses the within transformation to wipe out the $\mu_{i}^{\prime} s$ and then runs GLS estimation to account for the serial correlation in the remainder error. Premultiplying Equation (11) by $I_{n} \quad E_{T}^{\alpha}$, one gets

$$
\left(\begin{array}{ll}
I_{n} & E_{T}^{\alpha}
\end{array}\right) y^{*}=\left(\begin{array}{ll}
I_{n} & E_{T}^{\alpha}
\end{array}\right) x^{*} \beta+\left(\begin{array}{ll}
I_{n} & E_{T}^{\alpha}
\end{array}\right) v^{*}
$$

using $E_{T}^{\alpha} \iota_{T}^{\alpha}=0$. The least squares estimator of the transformed equation gives us the FE-GLS estimator, given by

$$
\hat{\beta}_{F E-G L S}=\frac{x^{* \prime}\left(I_{n}\right.}{\left.E_{T}^{\alpha}\right) y^{*}} .
$$

It is worth pointing out that the FE-GLS encompasses both the within and first-difference estimators. To see this, note that (i) if $\rho=0$, and there is no serial correlation in the remainder error, we have $C=I_{T}, x^{*}=x, \alpha=1, \iota_{T}^{\alpha}=\iota_{T}$ and hence $E_{T}^{\alpha}=E_{T}$, where $E_{T}=I_{T}-\bar{J}_{T}$ and $\bar{J}_{T}$ is 
a $T \times T$ matrix of $1 / T$. The FE-GLS estimator in Equation (17) reduces to the within estimator $\frac{x^{\prime}\left(I_{n}\right.}{\left.E_{T}\right) y}$.

(ii) Note also that $\bar{J}_{T}^{\alpha}$ can be rewritten as $\bar{J}_{T}^{\alpha}=l_{T}\left(l_{T}^{\prime} l_{T}\right)^{-1} l_{T}^{\prime}$, where $l_{T}=\sqrt{(1-\rho)} \iota_{T}^{\alpha^{\prime}}=$ $\sqrt{(1-\rho)}\left(\alpha, \iota_{T-1}^{\prime}\right)=\left(\sqrt{(1+\rho)}, \sqrt{(1-\rho)} \iota_{T-1}^{\prime}\right) \cdot\left(\right.$ If $\rho=1$, we have $l_{T}^{\prime}=\left(\sqrt{2}, 0_{T-1}^{\prime}\right)$ (and hence $\bar{J}_{T}^{\alpha}=\operatorname{diag}(1,0, \cdots, 0)$ and $E_{T}^{\alpha}=I_{T}-\bar{J}_{T}^{\alpha}=\left[\begin{array}{cc}0 & 0_{T-1}^{\prime} \\ 0_{T-1} & I_{T-1}\end{array}\right]$. Also, if $\rho=0, \mathbf{C}=\left[\begin{array}{c}0_{T}^{\prime} \\ D\end{array}\right](X$ with $D=\left[\begin{array}{cccccc}-1 & 1 & 0 & \cdots & 0 & 0 \\ \dot{y} & \vdots & \vdots & \ddots & \vdots & \vdots \\ 0 & 0 & 0 & -1 & 1 & 0 \\ 0 & 0 & 0 & 0 & -1 & 1\end{array}\right]$ which is the well-known first difference matrix. Hence $x^{*}=\left(f_{n}\left[\begin{array}{c}0_{T}^{\prime} \\ D\end{array}\right]\right)\left(x\right.$ and $\left[\begin{array}{ll}0_{T} & D^{\prime}\end{array}\right]\left(\left(\begin{array}{cc}0 & 0_{T-1}^{\prime} \\ D_{T-1} & I_{T-1}\end{array}\right]\left[\begin{array}{c}0_{T}^{\prime} \\ D\end{array}\right]\left(=D^{\prime} D\right.\right.$. The FE-GLS estimator in Equa-

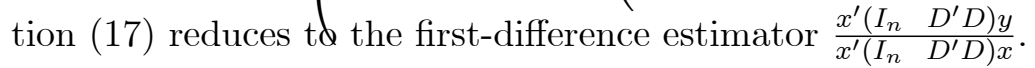

From Equation (17), we have

$$
\hat{\beta}_{F E-G L S}-\beta=\frac{F_{2}}{F_{1}},
$$

where $F_{1}=x_{i}^{* \prime} x_{i}^{*}-\frac{\left(\iota_{T}^{\alpha \prime} x_{i}^{*}\right)^{2}}{d^{2}}$ and $F_{2}=\frac{1}{n} \sum_{i}^{n}=1 x_{i}^{* \prime} u_{i}^{*}-\frac{\iota_{T}^{\alpha \prime} x_{i}^{*}}{n d^{2}} \sum_{i=1}^{n} \iota_{T}^{\alpha \prime} u_{i}^{*}$. It is easy to show that $\operatorname{Var}\left(\hat{\beta}_{F E-G L S}\right)=\frac{\sigma_{e}^{2}}{n F_{1}}$. Therefore,

$$
t_{F E-G L S}=\frac{\hat{\beta}_{F E-G L S}-\beta}{\sqrt{\left(\operatorname{Var}\left(\hat{\beta}_{F E-G L S}\right)\right.}}=\frac{F_{2} / F_{1}}{\sqrt{\sigma_{e}^{2} /\left(n F_{1}\right)}}=\frac{\sqrt{n} F_{2}}{\sqrt{\sigma_{e}^{2} F_{1}}} .
$$

Note that both the FE-GLS estimator of $\beta$ and its corresponding t-statistic do not depend on $\sigma_{\mu}^{2}$ or $\sigma_{\alpha}^{2}$. With a consistent estimator $\hat{\rho}$, the corresponding FE-FGLS estimator $\hat{\beta}_{F E-F G L S}$ is obtained by replacing $C$ and $E_{T}^{\alpha}$ by their corresponding estimators $\hat{C}$ and $\hat{E}_{T}^{\alpha}$. As suggested by Baltagi and $\mathrm{Li}$ (1991), an estimator of $\sigma_{e}^{2}$ can be obtained as $\hat{\sigma}_{e}^{2}=\frac{1}{n(T-1)} \hat{u}^{* \prime}\left(\begin{array}{ll}I_{n} & \hat{E}_{T}^{\alpha}\end{array}\right) \hat{\mu}^{*}$, where $\hat{u}^{*}$ is an $n T \times 1$ vector of OLS residuals from the Prais-Winsten transformed regression using $\hat{\rho}$. The corresponding t-statistic based on the FE-FGLS estimator can be obtained from equation (19). The asymptotic properties are summarized in the following Theorem:

Theorem 3 Assume $(n, T) \rightarrow \infty$,

1. When $|\rho|<1$, if $\hat{\rho} \stackrel{p}{\rightarrow} \rho$, we have

$$
t_{F E-F G L S} \stackrel{d}{\rightarrow} N(0,1) .
$$


2. When $\rho=1$, if $T(\hat{\rho}-1) \stackrel{p}{\rightarrow} \kappa$, we have

$$
\left.t_{F E-F G L S} \stackrel{d}{\rightarrow} N \quad 0, \frac{\left(\kappa^{2}-3 \kappa+3\right)\left(\kappa^{4}-10 \kappa^{3}+50 \kappa^{2}-120 \kappa+120\right)}{10\left(\kappa^{4}-\left(9 \kappa^{3}+33 \kappa^{2}-54 \kappa+36\right)\right.}\right) .
$$

Theorem 3 implies that we need $\kappa=0$ when $\rho=1$. Otherwise $t_{F E-F G L S}$ does not converge to a $N(0,1)$. Baltagi and Li (1991) suggest estimating $\rho$ using

$$
\hat{\rho}=\frac{\sum_{i=1}^{n} \sum_{t=2}^{T} \hat{\nu}_{i t} \hat{\nu}_{i, t-1}}{\sum_{i=1}^{n} \sum_{t=2}^{T} \hat{\nu}_{i, t-1}^{2}},
$$

where $\hat{\nu}_{i t}$ denotes the FE residual from equation (1) which is defined in Section 2. It can be obtained from a regression of $\hat{\nu}_{i t}$ on $\hat{\nu}_{i, t-1}$. The asymptotics for $\hat{\rho}$ are given in the following theorem:

Theorem 4 Assume $(n, T) \rightarrow \infty$,

1. If $|\rho|<1$,

2. If $\rho=1$,

$$
\sqrt{n T}\left(\hat{\rho}-\rho+\frac{1+\rho}{T}\right)(d \rightarrow
$$

$$
\sqrt{n} T\left(\not-1+\frac{3}{T}\right) \stackrel{d}{\rightarrow} N\left(0, \frac{51}{5}\right)(
$$

The asymptotic distribution of $\hat{\rho}$ in Theorem 4 is actually the same as Theorems 2 and 4 in Hahn and Kuersteiner (2002) that discuss a dynamic panel data model. Also, the result for the case where $\rho=1$ in Theorem 4 is the same as Theorem 2 in Kao (1999) which discusses the spurious panel data model. As we can see, the results from Theorem 4 : When $|\rho|<1$, there is a bias of $(1+\rho) / T$ in $\hat{\rho}$, but it still implies $\hat{\rho} \stackrel{p}{\rightarrow} \rho$ as $(n, T) \rightarrow \infty$. When $\rho=1, T(\hat{\rho}-1)$ does not converge to zero in probability if there are individual effects in the model. Substituting $\kappa=-3$ into Theorem 3 , one can verify that $\hat{\rho}$ suggested by Baltagi and Li (1991) leads to $t_{F E-F G L S} \stackrel{d}{\rightarrow} N\left(0, \frac{2989}{910}\right)$ when $\rho=1$. This limits the usefulness of the FE-GLS estimator when the error term is $I(1)$ and there are individual effects in the panel model. This difference is due to the fact that $\mu_{i}$ can not be consistently estimated when the error term is $I(1)$, see Kao and Emerson (2004). To achieve $t_{F E-F G L S} \stackrel{d}{\rightarrow} N(0,1)$, we need $\kappa=0$.

Therefore, when $|\rho|<1$, a bias-corrected estimator of $\rho$ is $\hat{\rho}+\frac{1+\hat{\rho}}{T}$. When $\rho=1$, a bias-corrected estimator of $\rho$ is $\hat{\rho}+\frac{3}{T}$. Combining the two cases, we suggest a bias-corrected estimator of $\rho$ as follows:

$$
\tilde{\rho}=\left\{\begin{array}{c}
\hat{\rho}+\frac{1+\hat{\rho}}{T} \quad \text { if } 1-\hat{\rho}>\frac{3}{T} \\
1 \quad \text { if } 1-\hat{\rho} \leq \frac{3}{T} \\
9
\end{array} .\right.
$$


The asymptotics for $\tilde{\rho}$ are given in the following theorem:

Theorem 5 Assume $(n, T) \rightarrow \infty$,

1. If $|\rho|<1$,

$$
\sqrt{n T}(\tilde{\rho}-\rho) \stackrel{d}{\rightarrow} N\left(0,1-\rho^{2}\right)
$$

2. If $\rho=1$,

$$
\sqrt{n} T(\tilde{\rho}-1) \stackrel{d}{\rightarrow} N\left(0, \frac{51}{5}\right)(
$$

Therefore, we have $t_{F E-F G L S} \stackrel{d}{\rightarrow} N(0,1)$ using $\tilde{\rho}$ for both $|\rho|<1$ and $\rho=1$.

In this section, we showed that the t-statistic based on FE-GLS is no longer robust if there are individual effects. Extra steps need to be taken to achieve the robustness when equation (1) includes individual effects.

\subsection{The Model Without Individual Effects}

Let us study the case where there are no individual effects, i.e., $\mu_{i}=0$ for all $i$. The variancecovariance matrix in equation (8) reduces to $\Phi=\sigma_{e}^{2}\left(\begin{array}{lll}I_{n} & \mathbf{A}\end{array}\right)$ and hence $\Phi^{-1}=\frac{1}{\sigma_{e}^{2}} I_{n} \quad \mathbf{A}^{-1}$. Equation (11) reduces to

$$
y^{*}=\delta \boldsymbol{\iota}_{n T}^{*}+\beta x^{*}+v^{*},
$$

where the variance-covariance matrix of the transformed disturbance is $E\left(v^{*} v^{* \prime}\right)=\sigma_{\varepsilon}^{2}\left(I_{n} \quad I_{T}\right)$. The least squares estimator of the transformed equation yields the GLS estimator:

$$
\hat{\beta}_{G L S}=\frac{x^{* \prime} M_{\iota_{n T}^{*}} y^{*}}{x^{* \prime} M_{\iota_{n T}^{*}} x^{*}},
$$

where $M_{\iota_{n T}^{*}}=I_{n T}-\boldsymbol{\iota}_{n T}^{*}\left(\boldsymbol{\iota}_{n T}^{* \prime} \boldsymbol{\iota}_{n T}^{*}\right) \boldsymbol{\iota}_{n T}^{* \prime}=I_{n T}-\bar{J}_{n} \quad \bar{J}_{T}^{\alpha}$ using the fact that $\boldsymbol{\iota}_{n T}^{*}=(1-\rho)\left(\begin{array}{ll}\boldsymbol{\iota}_{n} & \boldsymbol{\iota}_{T}^{\alpha}\end{array}\right)$. It is easy to see that $M_{\iota_{n T}^{*}} x^{*}=\left(\begin{array}{ll}I_{n T}-\bar{J}_{n} & \bar{J}_{T}^{\alpha}\end{array}\right)\left(\begin{array}{ll}\iota_{n} & x_{i}^{*}\end{array}\right)=\iota_{n} \quad\left(\begin{array}{ll}x_{i}^{*}-\bar{J}_{T}^{\alpha} x_{i}^{*}\end{array}\right)=\left(\begin{array}{ll}I_{n} & E_{T}^{\alpha}\end{array}\right) x^{*}$. This proves that $\hat{\beta}_{G L S}$ and $\hat{\beta}_{F E-G L S}$ are the same if there are no individual effects in the model. The t-statistic based on $\hat{\beta}_{G L S}$ is in turn the same as the one based on $\hat{\beta}_{F E-G L S}$ in equation (19), i.e.,

$$
t_{G L S}=\frac{\sqrt{n} F_{2}}{\sqrt{\sigma_{e}^{2} F_{1}}},
$$


where $F_{1}$ and $F_{2}$ are defined in Equation (18). Similar to equation (20) for the general model with individual effects, let

$$
\hat{\rho}=\frac{\sum_{i=1}^{n} \sum_{t=2}^{T} \hat{u}_{i t} \hat{u}_{i, t-1}}{\sum_{i=1}^{n} \sum_{t=2}^{T} \hat{u}_{i, t-1}^{2}},
$$

where $\hat{u}_{i t}$ is the OLS residual, i.e., $\hat{u}_{i t}=\left(y_{i t}-\overline{\bar{y}}\right)-\hat{\beta}_{O L S}(t-\bar{t})$ with $\overline{\bar{y}}=\frac{1}{n T} \sum_{i=1}^{n} \sum_{t=1}^{T} y_{i t}$ and $\bar{t}=\frac{1}{T} \sum_{t=1}^{T} t$. Define $\hat{u}^{*}$ as an $n T \times 1$ vector of OLS residuals from the Prais-Winsten transformed regression using $\hat{\rho}$. An estimator of $\sigma_{e}^{2}$ is $\hat{\sigma}_{e}^{2}=\frac{1}{n T} \hat{u}^{* \prime} \hat{u}^{*}$. Substituting $\hat{\rho}$ and $\hat{\sigma}_{e}^{2}$, the t-statistic corresponding to the FGLS estimator can be obtained from equation (23). The asymptotic properties are summarized in the following Theorem:

Theorem 6 Assume $(n, T) \rightarrow \infty$,

1. If $|\rho|<1$,

$$
\sqrt{n T}(\hat{\rho}-\rho) \stackrel{d}{\rightarrow} N\left(0,1-\rho^{2}\right)
$$

2. If $\rho=1$,

$$
T(\hat{\rho}-1) \stackrel{p}{\rightarrow} N(0,3) .
$$

Therefore, we have $t_{F G L S} \stackrel{d}{\rightarrow} N(0,1)$ using $\hat{\rho}$ for both $|\rho|<1$ and $\rho=1$.

Theorem 6 shows that $t_{F G L S}$ converges to $N(0,1)$, whether the error term is $I(0)$ or $I(1)$, when there are no individual effects in the model. This is an interesting result, i.e., the t-ratio based on FGLS effectively bridges the gap between the $I(0)$ and $I(1)$ error terms (if there are no individual effects in the model). This implies that inference on the slope parameter can be performed using the standard normal distribution if there are no individual effects. This is different from the pure time series model as in Perron and Yabu (2009) which requires a super-efficient estimate in order to achieve this goal. We know that this will change if there are individual effects in the model. This is the more likely case in panel data with heterogeneity across individuals.

\section{Monte Carlo Results}

This section reports the results of Monte Carlo experiments designed to investigate the finite sample properties of the FE-FGLS based $t_{F E-F G L S}$. The model is generated by

$$
y_{i t}=\alpha+\beta t+\mu_{i}+v_{i t}, \quad i=1, \ldots, n, \quad t=1, \ldots, T,
$$


with $\alpha=5, \beta=10, \mu_{i} \stackrel{i i d}{\sim} N(0,5)$, and $\nu_{i t}=\rho \nu_{i t-1}+e_{i t}$, with $\rho$ varying over the range $(0,0.2,0.4,0.6,0.8,0.9,1), v_{i 0}=0, e_{i t} \stackrel{i i d}{\sim} N\left(0, \sigma_{e}^{2}\right)$, and $\sigma_{e}^{2}=5$. The sample sizes $(n, T)$ are $(500,20),(500,50),(50,500),(10,100),(10,50),(50,10)$ and $(20,10)$, respectively. For each experiment, we perform 1,000 replications. For each replication we estimate the model using: (i) FD: first-difference ignoring serial correlation; (ii) FE: fixed-effects ignoring serial correlation; (iii) FEGLS: FE-GLS estimator using the true value of $\rho$; (iv) FE-FGLS 1 : FE-FGLS estimator using $\widehat{\rho}$ calculated by the method suggested in Baltagi and Li (1991); and (v) FE-FGLS 2 : FE-FGLS estimator using a bias-corrected estimator $\tilde{\rho}$. Table 1 reports the median, interquantile range and root mean squared error (RMSE) of estimators of $\hat{\rho}$ and $\tilde{\rho}$. Following Kelejian and Prucha (1999), RMSE is defined as $\left[\text { bias }^{2}+(I Q R / 1.35)^{2}\right]^{1 / 2}$, where bias is the difference between the median and the true parameter value and $I Q R$ is the interquantile range. That is $I Q R=c_{1}-c_{2}$, where $c_{1}$ and $c_{2}$ are the 0.75 and 0.25 quantiles respectively. As explained in Kelejian and Prucha (1999), these characteristics are closely related to the standard measures of bias and root mean squared error (RMSE) but, unlike these measures, are assured to exist. When the true $\rho$ is larger than 0.4, $\tilde{\rho}$ has smaller RMSE than $\hat{\rho}$. This is especially true when $\rho$ is close to 1 . For the first two $(n, T)$ combinations, Tables 2 and 3 report the size and power of the t-test for $H_{0}: \beta=0$ corresponding to each estimator of $\beta$. Tables 4 and 5 report the size-adjusted power. Several conclusions emerge from these results. For the FD estimator, if $\rho=1$, the size of the corresponding t-test is 0.064 when $n=500$ and $T=20$ and 0.053 when $n=500$ and $T=50$. However, the size is always zero for other values of $\rho$. For the FE estimator, if $\rho=0$, the size of the corresponding t-test is 0.057 when $n=500$ and $T=20$ and 0.043 when $n=500$ and $T=50$. The size increases with $\rho$. This verifies the asymptotic results in Theorem 1. The standard deviation increases with $\rho$ and decreases with T. This is consistent with the asymptotic results in Theorem 2. For the FE-FGLS estimators, the size of the corresponding t-test is too large for FE-FGLS 1 , especially if $\rho>0.4$. However, the

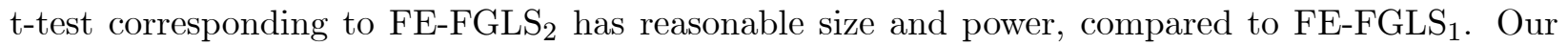
simulation results confirm the robustness of $t_{F E-F G L S_{2}}$ using the bias-corrected estimator $\tilde{\rho}$. For the other $(n, T)$ combinations, Tables 6 reports the size of the t-test for $H_{0}: \beta=0$ corresponding to each estimator. We can see that the results are robust to small samples and different ratios of $n / T$. 


\section{Conclusion}

In this paper, we discuss test of hypotheses in a linear time trend panel data model with serially correlated error component disturbances. The error term could be either stationary or nonstationary. We consider estimation and testing using the FE, FD and FE-GLS estimators. Different from the results in the pure time series case, the t-test based on FGLS always converges to $N(0,1)$ no matter whether the error term is $I(0)$ or $I(1)$, when there are no individual effects in the model. When there are individual effects in the model, the t-statistic based on FE-GLS is no longer robust. We suggest a bias-corrected estimator of $\rho$ to achieve robustness. We show that it performs well in Monte Carlo experiments and is recommended. While the focus of this paper is test of hypothesis in a simple linear trend panel data model with error components and serial correlation, it is important to extend this work to dynamic panel data models with cross-section dependence across the units. This should be the focus of future research.

\section{References}

[1] Baltagi, B. H. (2008), Econometric Analysis of Panel Data, Wiley.

[2] Baltagi, B.H. and Kao, C. (2000), "Nonstationary Panels, Cointegration in Panels and Dynamic Panels: A survey," Advances in Econometrics 15, 7-51.

[3] Baltagi, B. H., Kao, C., and Liu, L. (2008), "Asymptotic Properties of Estimators for the Linear Panel Regression Model with Random Individual Effects and Serially Correlated Errors: The Case of Stationary and Non-Stationary Regressors and Residuals," Econometrics Journal, 11, $554-572$.

[4] Baltagi, B. H., Kao, C., and Na, S. (2011), "Test of Hypotheses in Panel Data Models When the Regressor and Disturbances are Possibly Nonstationary," Advances in Statistical Analysis, 95, 329-350.

[5] Baltagi, B.H., and W. Krämer. (1997), "A Simple Linear Trend Model with Error Components, Problem 97.2.1," Econometric Theory 13, 463.

[6] Baltagi, B. H., and Li, Q. (1991), "A Transformation That Will Circumvent the Problem of Autocorrelation in an Error Component Model," Journal of Econometrics, 52, 371-380. 
[7] Breitung J., and Pesaran M.H. (2008), "Unit Roots and Cointegration in panels," Chapter 9 in L. Matyas and P. Sevestre (eds.) The Econometrics of Panel Data: Fundamentals and Recent Developments in Theory and Practice, Springer, Berlin, 279-322.

[8] Bunzel, H., and Vogelsang, T. J. (2005), "Powerful Trend Function Tests That are Robust to Strong Serial Correlation with an Application to the Prebish Singer Hypothesis," Journal of Business and Economic Statistics, 23, 381-394.

[9] Canjels, E., and Watson, M. W. (1997), "Estimating Deterministic Trends in the Presence of Serially Correlated Errors," Review of Economics and Statistics, 79, 184-200.

[10] Choi, I. (2006), "Nonstationary panels," Chapter 13 in T.C. Mills and K. Patterson (eds.), Palgrave Handbooks of Econometrics, Volume 1, pp. 511-539, Palgrave, Macmillan.

[11] Emerson, J. and Kao, C. (2000), "Testing for Structural Change of a Time Trend Regression in Panel Data," Center for Policy Research Working Papers 15, Center for Policy Research, Maxwell School, Syracuse University.

[12] Hahn, J., and Kuersteiner, G. (2002), "Asymptotically Unbiased Inference for a Dynamic Panel Model with Fixed Effects when Both n and T are Large," Econometrica, 70(4), 1639-1657.

[13] Kao, C. (1999), "Spurious Regression and Residual-Based Tests for Cointegration in Panel Data, " Journal of Econometrics, 90, 1-44.

[14] Kao, C., and Emerson, J. (2004), "On the Estimation of a Linear Time Trend Regression with a One-Way Error Component Model in the Presence of Serially Correlated Errors: Part I," Journal of Probability and Statistical Science, 2, 213-243.

[15] Kelejian, H. H., and Prucha, I. R. (1999), "A Generalized Moments Estimator for the Autoregressive Parameter in Spatial Model," International Economic Review, 40, 509-533.

[16] Phillips, P.C.B., and Moon, M. (1999), "Linear Regression Limit Theory for Nonstationary Panel Data," Econometrica 67, 1057-1111.

[17] Phillips, P.C.B., and Moon, M. (2000), "Nonstationary Panel Data Analysis: An overview of Some Recent Developments," Econometric Reviews 19, 263-286. 
[18] Perron, P., and Yabu, T. (2009), "Estimating Deterministic Trends with an Integrated or Stationary Noise Component," Journal of Econometrics, 151, 56-69.

[19] Roy, A., Falk, B., and Fuller, W. A. (2004), "Testing for Trend in the Presence of Autoregressive Error," Journal of the American Statistical Association, 99, 1082-1091.

[20] Vogelsang, T. J. (1998), "Trend Function Hypothesis Testing in the Presence of Serial Correlation," Econometrica, 66, 123-148.

[21] Vogelsang, T. J., and Fomby, T. B. (2002), "The Application of Size Robust Trend Analysis to Global Warming Temperature Series," Journal of Climate, 15, 117-123. 
Table 1: Median, IQR and RMSE of Estimators of $\rho$

\begin{tabular}{|c|c|c|c|c|c|c|c|c|}
\hline \multirow{2}{*}{$\mathrm{n}$} & \multirow[b]{2}{*}{$\mathrm{T}$} & \multirow[b]{2}{*}{$\rho$} & \multicolumn{3}{|c|}{$\rho$} & \\
\hline & & & Median & IQR & RMSE & Median & IQR & RMSE \\
\hline \multirow[t]{7}{*}{500} & 20 & 0 & 0.000 & 0.013 & 0.010 & 0.050 & 0.014 & 0.051 \\
\hline & & 0.2 & 0.178 & 0.013 & 0.024 & 0.237 & 0.014 & 0.039 \\
\hline & & 0.4 & 0.354 & 0.013 & 0.047 & 0.422 & 0.013 & 0.024 \\
\hline & & 0.6 & 0.525 & 0.012 & 0.075 & 0.602 & 0.012 & 0.009 \\
\hline & & 0.8 & 0.686 & 0.010 & 0.115 & 0.770 & 0.011 & 0.031 \\
\hline & & 0.9 & 0.760 & 0.009 & 0.140 & 0.848 & 0.009 & 0.053 \\
\hline & & 1 & 0.857 & 0.007 & 0.143 & 1.000 & 0.000 & 0.000 \\
\hline \multirow[t]{7}{*}{500} & 50 & 0 & 0.000 & 0.008 & 0.006 & 0.020 & 0.008 & 0.021 \\
\hline & & 0.2 & 0.192 & 0.008 & 0.010 & 0.216 & 0.008 & 0.017 \\
\hline & & 0.4 & 0.382 & 0.008 & 0.018 & 0.410 & 0.008 & 0.012 \\
\hline & & 0.6 & 0.572 & 0.007 & 0.029 & 0.603 & 0.007 & 0.006 \\
\hline & & 0.8 & 0.758 & 0.006 & 0.042 & 0.793 & 0.006 & 0.008 \\
\hline & & 0.9 & 0.847 & 0.005 & 0.053 & 0.884 & 0.005 & 0.016 \\
\hline & & 1 & 0.941 & 0.003 & 0.059 & 1.000 & 0.022 & 0.016 \\
\hline \multirow[t]{7}{*}{50} & 500 & 0 & 0.000 & 0.009 & 0.006 & 0.002 & 0.009 & 0.007 \\
\hline & & 0.2 & 0.199 & 0.008 & 0.006 & 0.202 & 0.008 & 0.006 \\
\hline & & 0.4 & 0.398 & 0.008 & 0.006 & 0.401 & 0.008 & 0.006 \\
\hline & & 0.6 & 0.597 & 0.007 & 0.006 & 0.600 & 0.007 & 0.005 \\
\hline & & 0.8 & 0.796 & 0.005 & 0.006 & 0.800 & 0.005 & 0.004 \\
\hline & & 0.9 & 0.895 & 0.004 & 0.006 & 0.899 & 0.004 & 0.003 \\
\hline & & 1 & 0.994 & 0.001 & 0.006 & 0.998 & 0.003 & 0.003 \\
\hline \multirow[t]{7}{*}{10} & 100 & 0 & -0.001 & 0.041 & 0.030 & 0.009 & 0.041 & 0.032 \\
\hline & & 0.2 & 0.195 & 0.041 & 0.031 & 0.207 & 0.041 & 0.031 \\
\hline & & 0.4 & 0.389 & 0.038 & 0.030 & 0.403 & 0.038 & 0.029 \\
\hline & & 0.6 & 0.585 & 0.034 & 0.029 & 0.601 & 0.034 & 0.025 \\
\hline & & 0.8 & 0.778 & 0.027 & 0.030 & 0.796 & 0.027 & 0.020 \\
\hline & & 0.9 & 0.873 & 0.021 & 0.031 & 0.892 & 0.022 & 0.018 \\
\hline & & 1 & 0.967 & 0.013 & 0.034 & 0.987 & 0.020 & 0.020 \\
\hline \multirow[t]{7}{*}{10} & 50 & 0 & -0.004 & 0.057 & 0.042 & 0.016 & 0.058 & 0.046 \\
\hline & & 0.2 & 0.187 & 0.057 & 0.044 & 0.210 & 0.058 & 0.044 \\
\hline & & 0.4 & 0.378 & 0.054 & 0.046 & 0.405 & 0.055 & 0.041 \\
\hline & & 0.6 & 0.566 & 0.050 & 0.050 & 0.597 & 0.051 & 0.038 \\
\hline & & 0.8 & 0.753 & 0.041 & 0.056 & 0.788 & 0.042 & 0.033 \\
\hline & & 0.9 & 0.841 & 0.037 & 0.064 & 0.878 & 0.037 & 0.035 \\
\hline & & 1 & 0.934 & 0.026 & 0.069 & 0.973 & 0.041 & 0.041 \\
\hline \multirow[t]{7}{*}{50} & 10 & 0 & -0.005 & 0.057 & 0.042 & 0.095 & 0.062 & 0.106 \\
\hline & & 0.2 & 0.152 & 0.060 & 0.065 & 0.268 & 0.066 & 0.083 \\
\hline & & 0.4 & 0.302 & 0.058 & 0.107 & 0.433 & 0.064 & 0.057 \\
\hline & & 0.6 & 0.440 & 0.055 & 0.165 & 0.584 & 0.061 & 0.048 \\
\hline & & 0.8 & 0.565 & 0.052 & 0.238 & 0.722 & 0.057 & 0.089 \\
\hline & & 0.9 & 0.632 & 0.050 & 0.271 & 0.795 & 0.055 & 0.113 \\
\hline & & 1 & 0.722 & 0.041 & 0.279 & 1.000 & 0.000 & 0.000 \\
\hline \multirow[t]{7}{*}{20} & 10 & 0 & -0.003 & 0.088 & 0.065 & 0.097 & 0.097 & 0.120 \\
\hline & & 0.2 & 0.152 & 0.090 & 0.083 & 0.267 & 0.099 & 0.100 \\
\hline & & 0.4 & 0.299 & 0.089 & 0.120 & 0.429 & 0.098 & 0.078 \\
\hline & & 0.6 & 0.435 & 0.083 & 0.177 & 0.578 & 0.092 & 0.071 \\
\hline & & 0.8 & 0.559 & 0.079 & 0.248 & 0.714 & 0.087 & 0.107 \\
\hline & & 0.9 & 0.627 & 0.07516 & $\begin{array}{ll}6 & 0.279\end{array}$ & 0.789 & 0.083 & 0.127 \\
\hline & & 1 & 0.716 & 0.068 & 0.288 & 1.000 & 0.151 & 0.112 \\
\hline
\end{tabular}


Table 2: Size and Power of the t-test for $H_{0}: \beta=0(n=500, T=20)$

\begin{tabular}{rr|ccccc}
\hline$\rho$ & $\beta$ & FD & FE & FE-GLS & FE-FGLS & FE-FGLS \\
\hline 0 & 0.00 & 0.000 & 0.057 & 0.054 & 0.053 & 0.045 \\
0 & 0.02 & 0.000 & 1.000 & 1.000 & 1.000 & 0.999 \\
0 & 0.04 & 0.000 & 1.000 & 1.000 & 1.000 & 1.000 \\
0 & 0.06 & 0.335 & 1.000 & 1.000 & 1.000 & 1.000 \\
0 & 0.08 & 0.986 & 1.000 & 1.000 & 1.000 & 1.000 \\
0 & 0.10 & 1.000 & 1.000 & 1.000 & 1.000 & 1.000 \\
\hline 0.2 & 0.00 & 0.000 & 0.105 & 0.054 & 0.059 & 0.046 \\
0.2 & 0.02 & 0.000 & 0.996 & 0.969 & 0.972 & 0.960 \\
0.2 & 0.04 & 0.003 & 1.000 & 1.000 & 1.000 & 1.000 \\
0.2 & 0.06 & 0.643 & 1.000 & 1.000 & 1.000 & 1.000 \\
0.2 & 0.08 & 0.997 & 1.000 & 1.000 & 1.000 & 1.000 \\
0.2 & 0.10 & 1.000 & 1.000 & 1.000 & 1.000 & 1.000 \\
\hline 0.4 & 0.00 & 0.000 & 0.174 & 0.054 & 0.067 & 0.049 \\
0.4 & 0.02 & 0.000 & 0.979 & 0.833 & 0.865 & 0.810 \\
0.4 & 0.04 & 0.040 & 1.000 & 1.000 & 1.000 & 1.000 \\
0.4 & 0.06 & 0.815 & 1.000 & 1.000 & 1.000 & 1.000 \\
0.4 & 0.08 & 0.999 & 1.000 & 1.000 & 1.000 & 1.000 \\
0.4 & 0.10 & 1.000 & 1.000 & 1.000 & 1.000 & 1.000 \\
\hline 0.6 & 0.00 & 0.000 & 0.283 & 0.054 & 0.087 & 0.053 \\
0.6 & 0.02 & 0.000 & 0.935 & 0.506 & 0.625 & 0.504 \\
0.6 & 0.04 & 0.139 & 1.000 & 0.969 & 0.988 & 0.964 \\
0.6 & 0.06 & 0.894 & 1.000 & 1.000 & 1.000 & 1.000 \\
0.6 & 0.08 & 1.000 & 1.000 & 1.000 & 1.000 & 1.000 \\
0.6 & 0.10 & 1.000 & 1.000 & 1.000 & 1.000 & 1.000 \\
\hline 0.8 & 0.00 & 0.000 & 0.388 & 0.054 & 0.117 & 0.071 \\
0.8 & 0.02 & 0.002 & 0.812 & 0.159 & 0.378 & 0.219 \\
0.8 & 0.04 & 0.248 & 0.995 & 0.506 & 0.822 & 0.611 \\
0.8 & 0.06 & 0.909 & 1.000 & 0.833 & 0.979 & 0.912 \\
0.8 & 0.08 & 0.999 & 1.000 & 0.969 & 1.000 & 0.987 \\
0.8 & 0.10 & 1.000 & 1.000 & 1.000 & 1.000 & 1.000 \\
\hline 0.9 & 0.00 & 0.000 & 0.455 & 0.054 & 0.139 & 0.084 \\
0.9 & 0.02 & 0.025 & 0.741 & 0.091 & 0.289 & 0.146 \\
0.9 & 0.04 & 0.329 & 0.972 & 0.159 & 0.624 & 0.342 \\
0.9 & 0.06 & 0.860 & 1.000 & 0.314 & 0.887 & 0.609 \\
0.9 & 0.08 & 0.997 & 1.000 & 0.506 & 0.980 & 0.828 \\
0.9 & 0.10 & 1.000 & 1.000 & 0.671 & 1.000 & 0.946 \\
\hline 1 & 0.00 & 0.064 & 0.577 & 0.064 & 0.284 & 0.078 \\
1 & 0.02 & 0.146 & 0.681 & 0.146 & 0.423 & 0.159 \\
1 & 0.04 & 0.410 & 0.855 & 0.410 & 0.696 & 0.419 \\
1 & 0.06 & 0.721 & 0.952 & 0.721 & 0.904 & 0.732 \\
1 & 0.08 & 0.921 & 0.996 & 0.921 & 0.988 & 0.930 \\
1 & 0.10 & 0.989 & 1.000 & 0.989 & 0.998 & 0.990 \\
\hline \hline & & & & & &
\end{tabular}


Table 3: Size and Power of the t-test for $H_{0}: \beta=0(n=500, T=50)$

\begin{tabular}{rr|ccccc}
\hline$\rho$ & $\beta$ & FD & FE & FE-GLS & FE-FGLS & FE-FGLS $_{2}$ \\
\hline 0 & 0.00 & 0.000 & 0.043 & 0.052 & 0.052 & 0.050 \\
0 & 0.02 & 0.000 & 1.000 & 1.000 & 1.000 & 1.000 \\
0 & 0.04 & 0.569 & 1.000 & 1.000 & 1.000 & 1.000 \\
0 & 0.06 & 1.000 & 1.000 & 1.000 & 1.000 & 1.000 \\
\hline 0.2 & 0.00 & 0.000 & 0.108 & 0.052 & 0.054 & 0.050 \\
0.2 & 0.02 & 0.000 & 1.000 & 1.000 & 1.000 & 1.000 \\
0.2 & 0.04 & 0.900 & 1.000 & 1.000 & 1.000 & 1.000 \\
0.2 & 0.06 & 1.000 & 1.000 & 1.000 & 1.000 & 1.000 \\
\hline 0.4 & 0.00 & 0.000 & 0.194 & 0.052 & 0.056 & 0.050 \\
0.4 & 0.02 & 0.000 & 1.000 & 1.000 & 1.000 & 1.000 \\
0.4 & 0.04 & 0.990 & 1.000 & 1.000 & 1.000 & 1.000 \\
0.4 & 0.06 & 1.000 & 1.000 & 1.000 & 1.000 & 1.000 \\
\hline 0.6 & 0.00 & 0.000 & 0.315 & 0.052 & 0.072 & 0.052 \\
0.6 & 0.02 & 0.000 & 1.000 & 1.000 & 1.000 & 1.000 \\
0.6 & 0.04 & 0.998 & 1.000 & 1.000 & 1.000 & 1.000 \\
0.6 & 0.06 & 1.000 & 1.000 & 1.000 & 1.000 & 1.000 \\
\hline 0.8 & 0.00 & 0.000 & 0.486 & 0.052 & 0.098 & 0.056 \\
0.8 & 0.02 & 0.010 & 1.000 & 0.973 & 0.994 & 0.977 \\
0.8 & 0.04 & 0.997 & 1.000 & 1.000 & 1.000 & 1.000 \\
0.8 & 0.06 & 1.000 & 1.000 & 1.000 & 1.000 & 1.000 \\
\hline 0.9 & 0.00 & 0.000 & 0.590 & 0.052 & 0.126 & 0.074 \\
0.9 & 0.02 & 0.044 & 0.993 & 0.502 & 0.788 & 0.609 \\
0.9 & 0.04 & 0.988 & 1.000 & 0.973 & 1.000 & 0.996 \\
0.9 & 0.06 & 1.000 & 1.000 & 1.000 & 1.000 & 1.000 \\
\hline 1 & 0.00 & 0.053 & 0.706 & 0.053 & 0.250 & 0.072 \\
1 & 0.02 & 0.271 & 0.854 & 0.271 & 0.605 & 0.328 \\
1 & 0.04 & 0.794 & 0.989 & 0.794 & 0.954 & 0.832 \\
1 & 0.06 & 0.988 & 1.000 & 0.988 & 0.999 & 0.992 \\
1 & 0.08 & 1.000 & 1.000 & 1.000 & 1.000 & 1.000 \\
\hline \hline & & & & & & \\
\hline
\end{tabular}

Notes: Cases with power of 1.000 have been omitted after their first occurrence. 
Table 4: Size-adjusted Power of the t-test for $H_{0}: \beta=0(n=500, T=20)$

\begin{tabular}{rr|ccccc}
\hline$\rho$ & $\beta$ & FD & FE & FE-GLS & FE-FGLS & FE-FGLS $_{2}$ \\
\hline 0 & 0.02 & 0.780 & 1.000 & 0.999 & 0.999 & 0.999 \\
0 & 0.04 & 0.999 & 1.000 & 1.000 & 1.000 & 1.000 \\
0 & 0.06 & 1.000 & 1.000 & 1.000 & 1.000 & 1.000 \\
\hline 0.2 & 0.02 & 0.801 & 0.986 & 0.961 & 0.962 & 0.961 \\
0.2 & 0.04 & 0.999 & 1.000 & 1.000 & 1.000 & 1.000 \\
0.2 & 0.06 & 1.000 & 1.000 & 1.000 & 1.000 & 1.000 \\
\hline 0.4 & 0.02 & 0.780 & 0.930 & 0.815 & 0.826 & 0.811 \\
0.4 & 0.04 & 0.998 & 1.000 & 1.000 & 1.000 & 1.000 \\
0.4 & 0.06 & 1.000 & 1.000 & 1.000 & 1.000 & 1.000 \\
\hline 0.6 & 0.02 & 0.700 & 0.716 & 0.472 & 0.525 & 0.480 \\
0.6 & 0.04 & 0.996 & 0.998 & 0.961 & 0.975 & 0.962 \\
0.6 & 0.06 & 1.000 & 1.000 & 1.000 & 1.000 & 1.000 \\
\hline 0.8 & 0.02 & 0.500 & 0.409 & 0.136 & 0.231 & 0.171 \\
0.8 & 0.04 & 0.968 & 0.936 & 0.472 & 0.679 & 0.551 \\
0.8 & 0.06 & 1.000 & 1.000 & 0.815 & 0.960 & 0.873 \\
0.8 & 0.08 & 1.000 & 1.000 & 0.961 & 1.000 & 0.980 \\
0.8 & 0.1 & 1.000 & 1.000 & 0.999 & 1.000 & 1.000 \\
\hline 0.9 & 0.02 & 0.308 & 0.234 & 0.073 & 0.131 & 0.099 \\
0.9 & 0.04 & 0.852 & 0.734 & 0.136 & 0.402 & 0.268 \\
0.9 & 0.06 & 0.997 & 0.975 & 0.289 & 0.740 & 0.512 \\
0.9 & 0.08 & 1.000 & 1.000 & 0.472 & 0.946 & 0.752 \\
0.9 & 0.1 & 1.000 & 1.000 & 0.647 & 0.992 & 0.920 \\
\hline 1 & 0.02 & 0.117 & 0.095 & 0.117 & 0.115 & 0.111 \\
1 & 0.04 & 0.373 & 0.293 & 0.373 & 0.363 & 0.356 \\
1 & 0.06 & 0.687 & 0.598 & 0.687 & 0.674 & 0.658 \\
1 & 0.08 & 0.900 & 0.844 & 0.900 & 0.892 & 0.891 \\
1 & 0.1 & 0.983 & 0.957 & 0.983 & 0.982 & 0.983 \\
\hline \hline
\end{tabular}

Notes: Cases with power of 1.000 have been omitted after their first occurrence. 
Table 5: Size-adjusted Power of the t-test for $H_{0}: \beta=0(n=500, T=50)$

\begin{tabular}{rr|ccccc}
\hline$\rho$ & $\beta$ & FD & FE & FE-GLS & FE-FGLS & FE-FGLS $_{2}$ \\
\hline 0 & 0.02 & 1.000 & 1.000 & 1.000 & 1.000 & 1.000 \\
\hline 0.2 & 0.02 & 1.000 & 1.000 & 1.000 & 1.000 & 1.000 \\
\hline 0.4 & 0.02 & 1.000 & 1.000 & 1.000 & 1.000 & 1.000 \\
\hline 0.6 & 0.02 & 1.000 & 1.000 & 1.000 & 1.000 & 1.000 \\
\hline 0.8 & 0.02 & 1.000 & 0.998 & 0.970 & 0.984 & 0.975 \\
0.8 & 0.04 & 1.000 & 1.000 & 1.000 & 1.000 & 1.000 \\
\hline 0.9 & 0.02 & 0.979 & 0.884 & 0.492 & 0.652 & 0.554 \\
0.9 & 0.04 & 1.000 & 1.000 & 0.970 & 0.998 & 0.989 \\
0.9 & 0.06 & 1.000 & 1.000 & 1.000 & 1.000 & 1.000 \\
\hline 1 & 0.02 & 0.265 & 0.248 & 0.265 & 0.254 & 0.260 \\
1 & 0.04 & 0.792 & 0.734 & 0.792 & 0.782 & 0.776 \\
1 & 0.06 & 0.987 & 0.973 & 0.987 & 0.987 & 0.986 \\
1 & 0.08 & 1.000 & 1.000 & 1.000 & 0.999 & 0.999 \\
1 & 0.1 & 1.000 & 1.000 & 1.000 & 1.000 & 1.000 \\
\hline \hline
\end{tabular}

Notes: Cases with power of 1.000 have been omitted after their first occurrence. 
Table 6: Size of the t-test for $H_{0}: \beta=0$

\begin{tabular}{|c|c|c|c|c|c|c|c|}
\hline$n$ & $T$ & $\rho$ & FD & $\mathrm{FE}$ & FE-GLS & FE-FGLS $_{1}$ & $\mathrm{FE} \mathrm{FGLS}_{2}$ \\
\hline \multirow[t]{7}{*}{50} & 500 & 0 & 0.000 & 0.059 & 0.060 & 0.056 & 0.057 \\
\hline & & 0.2 & 0.000 & 0.112 & 0.060 & 0.057 & 0.057 \\
\hline & & 0.4 & 0.000 & 0.192 & 0.060 & 0.057 & 0.057 \\
\hline & & 0.6 & 0.000 & 0.305 & 0.060 & 0.059 & 0.057 \\
\hline & & 0.8 & 0.000 & 0.507 & 0.060 & 0.060 & 0.059 \\
\hline & & 0.9 & 0.000 & 0.660 & 0.060 & 0.067 & 0.060 \\
\hline & & 1 & 0.054 & 0.910 & 0.054 & 0.168 & 0.064 \\
\hline \multirow[t]{7}{*}{10} & 100 & 0 & 0.000 & 0.047 & 0.050 & 0.044 & 0.046 \\
\hline & & 0.2 & 0.000 & 0.096 & 0.050 & 0.052 & 0.050 \\
\hline & & 0.4 & 0.000 & 0.175 & 0.050 & 0.053 & 0.052 \\
\hline & & 0.6 & 0.000 & 0.296 & 0.050 & 0.054 & 0.053 \\
\hline & & 0.8 & 0.000 & 0.465 & 0.050 & 0.070 & 0.056 \\
\hline & & 0.9 & 0.000 & 0.609 & 0.050 & 0.097 & 0.067 \\
\hline & & 1 & 0.064 & 0.802 & 0.064 & 0.198 & 0.083 \\
\hline \multirow[t]{7}{*}{10} & 50 & 0 & 0.000 & 0.049 & 0.047 & 0.041 & 0.037 \\
\hline & & 0.2 & 0.000 & 0.118 & 0.047 & 0.055 & 0.045 \\
\hline & & 0.4 & 0.000 & 0.209 & 0.047 & 0.063 & 0.049 \\
\hline & & 0.6 & 0.000 & 0.334 & 0.047 & 0.072 & 0.055 \\
\hline & & 0.8 & 0.000 & 0.476 & 0.047 & 0.111 & 0.067 \\
\hline & & 0.9 & 0.000 & 0.586 & 0.047 & 0.164 & 0.088 \\
\hline & & 1 & 0.047 & 0.753 & 0.047 & 0.215 & 0.087 \\
\hline \multirow[t]{7}{*}{50} & 10 & 0 & 0.000 & 0.051 & 0.042 & 0.036 & 0.025 \\
\hline & & 0.2 & 0.000 & 0.095 & 0.042 & 0.053 & 0.029 \\
\hline & & 0.4 & 0.000 & 0.153 & 0.042 & 0.067 & 0.037 \\
\hline & & 0.6 & 0.000 & 0.223 & 0.042 & 0.091 & 0.047 \\
\hline & & 0.8 & 0.000 & 0.297 & 0.042 & 0.126 & 0.057 \\
\hline & & 0.9 & 0.002 & 0.335 & 0.042 & 0.133 & 0.056 \\
\hline & & 1 & 0.037 & 0.424 & 0.037 & 0.131 & 0.044 \\
\hline \multirow[t]{7}{*}{20} & 10 & 0 & 0.000 & 0.050 & 0.041 & 0.035 & 0.030 \\
\hline & & 0.2 & 0.000 & 0.092 & 0.041 & 0.047 & 0.029 \\
\hline & & 0.4 & 0.000 & 0.139 & 0.041 & 0.063 & 0.035 \\
\hline & & 0.6 & 0.000 & 0.203 & 0.041 & 0.082 & 0.044 \\
\hline & & 0.8 & 0.002 & 0.278 & 0.041 & 0.116 & 0.058 \\
\hline & & 0.9 & 0.008 & 0.321 & 0.041 & 0.126 & 0.062 \\
\hline & & 1 & 0.053 & 0.399 & 0.053 & 0.119 & 0.073 \\
\hline
\end{tabular}




\section{Appendix}

\section{A Proof of Theorem 1}

Proof. The proof of (1) is a textbook result and hence omitted here.

Consider (2). Recall $\hat{\sigma}_{v}^{2}=\frac{1}{n(T-1)-1} \sum_{i=1}^{n} \sum_{t=2}^{T} \hat{\nu}_{i t}^{2}$, where $\hat{\nu}_{i t}=\left(y_{i t}-\bar{y}_{i .}\right)-\hat{\beta}_{F E}(t-\bar{t})=$ $\left(v_{i t}-\bar{v}_{i .}\right)-\left(\hat{\beta}_{F E}-\beta\right)(t-\bar{t})$. Hence

$$
\begin{aligned}
\frac{1}{T} \hat{\sigma}_{v}^{2} \simeq & \frac{1}{n T^{2}} \sum_{i=1}^{n} \sum_{t=2}^{T} \hat{\nu}_{i t}^{2}=\frac{1}{n T^{2}} \sum_{i=1}^{n} \sum_{t=2}^{T}\left(v_{i t}-\bar{v}_{i .}\right)^{2}+\frac{1}{n T^{2}}\left[\sqrt{n T}\left(\hat{\beta}_{F E}-\beta\right)\right]^{2}\left\{\frac{1}{n T} \sum_{i=1}^{n} \sum_{t=2}^{T}(t-\bar{t})^{2}\right\} \\
& -\frac{2}{n}\left[\sqrt{n T}\left(\hat{\beta}_{F E}-\beta\right)\right]\left\{\left(\frac{1}{\sqrt{n} T^{5 / 2}} \sum_{i=1}^{n} \sum_{t=2}^{T}\left(v_{i t}-\bar{v}_{i .}\right)(t-\bar{t})\right\} .\right.
\end{aligned}
$$

Notice that

by equation (C3) in Kao (1999),

$$
\frac{1}{n T^{2}} \sum_{i=1}^{n} \sum_{t=2}^{T}\left(v_{i t}-\bar{v}_{i .}\right)^{2} \stackrel{p}{\rightarrow} \frac{\sigma_{e}^{2}}{6}
$$

$$
\sqrt{n T}\left(\hat{\beta}_{F E}-\beta\right) \stackrel{d}{\rightarrow} N\left(0, \frac{6}{5} \sigma_{e}^{2}\right)
$$

by Theorem 4 in Kao and Emerson (2004), and

$$
\frac{1}{\sqrt{n} T^{5 / 2}} \sum_{i=1}^{n} \sum_{t=2}^{T}\left(v_{i t}-\bar{v}_{i .}\right)(t-\bar{t}) \stackrel{d}{\rightarrow} 3 N\left(\oint, \frac{\sigma_{e}^{2}}{120}\right)(
$$

by an equation on page 23 in Kao and Emerson (2004).

Hence we have

and therefore

$$
\begin{gathered}
n T^{2} \operatorname{Var}\left(\hat{\beta}_{F E}\right)=\frac{\frac{1}{T} \hat{\sigma}_{v}^{2} \stackrel{p}{\rightarrow} \frac{\sigma_{e}^{2}}{6},}{\frac{1}{n T^{3} \sum_{i}^{n} f_{1} \sum_{t=1}^{T}(t-\bar{t})^{2}} \stackrel{p}{\rightarrow} \frac{\sigma_{e}^{2} / 6}{1 / 12}=2 \sigma_{e}^{2}} \\
\frac{1}{\sqrt{T}} t_{F E}=\frac{\sqrt{n T}\left(\hat{\beta}_{F E}-\beta\right)}{\sqrt{\left(T^{2} \operatorname{Var}\left(\widehat{\beta}_{F E}\right)\right.}} \stackrel{d}{\rightarrow} \frac{N\left(\phi, \frac{6}{5} \sigma_{e}^{2}\right)}{\sqrt{2 \sigma_{e}^{2}}}=N\left(0, \frac{3}{5}\right) .
\end{gathered}
$$




\section{B Proof of Theorem 2}

Proof. Consider (1). Recall $\Delta y_{i t}=\beta+\Delta \nu_{i t}$ and hence $\Delta y_{i t}-\hat{\beta}_{F D}=\Delta \nu_{i t}-\left(\hat{\beta}_{F D}-\beta\right) \cdot(\mathrm{We}$
have

$$
\begin{aligned}
\hat{\sigma}_{e}^{2} & \simeq \frac{1}{n T} \sum_{i=1}^{n} \sum_{t=2}^{T}\left(\not y_{i t}-\hat{\beta}_{F D}\right)^{2}=\frac{1}{n T} \sum_{i=1}^{n} \sum_{t=2}^{T}\left[\Delta \nu_{i t}-\left(\hat{\beta}_{F D}-\beta\right)\right]^{2} \\
& =\frac{1}{n T} \sum_{i=1}^{n} \sum_{t=2}^{T}\left(\Delta \nu_{i t}\right)^{2}+\frac{1}{n T^{2}}\left[\sqrt{n} T\left(\hat{\beta}_{F D}-\beta\right)\right]^{2}-\frac{2}{\sqrt{n} T}\left[\sqrt{n} T\left(\hat{\beta}_{F D}-\beta\right)\right]\left(\frac{1}{n T} \sum_{i=1}^{n} \sum_{t=2}^{T} \Delta \nu_{i t}\right)(
\end{aligned}
$$

Notice that

$$
\begin{aligned}
& \frac{1}{n T} \sum_{i=1}^{n} \sum_{t=2}^{T}\left(\Delta \nu_{i t}\right)^{2}=(\rho-1)^{2} \frac{1}{n T} \sum_{i=1}^{n} \sum_{t=2}^{T} \nu_{i, t-1}^{2}+\frac{1}{n T} \sum_{i=1}^{n} \sum_{t=2}^{T} \oint_{i t}^{2}+2(\rho-1) \frac{1}{n T} \sum_{i=1}^{n} \sum_{t=2}^{T} \nu_{i, t-1} e_{i t} \\
& \stackrel{p}{\rightarrow}(\rho-1)^{2} \frac{\sigma_{e}^{2}}{1-\rho^{2}}+\sigma_{e}^{2}+0=\frac{2 \sigma_{e}^{2}}{1+\rho}, \\
& \frac{1}{n T} \sum_{i=1}^{n} \sum_{t=2}^{T} \Delta \nu_{i t} \stackrel{p}{\rightarrow} 0
\end{aligned}
$$

and

$$
\sqrt{n} T\left(\hat{\beta}_{F D}-\beta\right) \stackrel{d}{\rightarrow} N\left(0, \frac{2 \sigma_{e}^{2}}{1-\rho^{2}}\right)
$$

by Theorem 3 in Kao and Emerson (2004).

Hence we have

$$
n T \operatorname{Var}\left(\hat{\beta}_{F D}\right)\left(=\hat{\sigma}_{e}^{2} \stackrel{p}{\rightarrow} \frac{2 \sigma_{e}^{2}}{1+\rho},\right.
$$

and

$$
\sqrt{T} t_{F D}=\frac{\sqrt{n} T\left(\hat{\beta}_{F D}-\beta_{0}\right)}{\sqrt{\ell T \operatorname{Var}\left(\hat{\beta}_{F D}\right)}} \stackrel{d}{\rightarrow} \frac{N\left(0, \frac{2}{1-\rho^{2}} \sigma_{e}^{2}\right)}{\sqrt{\frac{2 \sigma_{e}^{2}}{1+\rho}}}=N\left(0, \frac{1}{1-\rho}\right) .
$$

Part (2) can be shown easily following Kao and Emerson (2004) and hence omitted. 


\section{Lemma 1}

$$
\begin{aligned}
& \mathbf{x}_{i}^{* \prime} \mathbf{x}_{i}^{*}=(1-\hat{\rho})^{2} \sum_{t=2}^{T} t^{2}+2 \hat{\rho}(1-\hat{\rho}) \sum_{t=2}^{T}\left(+\hat{\rho}^{2}(T-1)+\left(1-\hat{\rho}^{2}\right),\right. \\
& \hat{\iota}_{T}^{\alpha \prime} \mathbf{x}_{i}^{*}=(1-\hat{\rho}) \sum_{t=2}^{T}(+\hat{\rho}(T-1)+(1+\hat{\rho}), \\
& \mathbf{x}_{i}^{* \prime} \mathbf{v}_{i}^{*}=\left(1-\hat{\rho}^{2}\right) \psi_{i 1}+(1-\hat{\rho})(\rho-\hat{\rho}) \sum_{t=2}^{T} t \nu_{i, t-1}+\hat{\rho}(\rho-\hat{\rho}) \sum_{t=2}^{T} \nu_{i, t-1}+(1-\hat{\rho}) \sum_{t=2}^{T} t e_{i t}+\hat{\rho} \sum_{t=2}^{T} \xi_{i t}, \\
& \hat{\iota}_{T}^{\alpha \prime} \mathbf{v}_{i}^{*}=(1+\hat{\rho}) v_{i 1}+(\rho-\hat{\rho}) \sum_{t=2}^{T} \nu_{i, t-1}+\sum_{t=2}^{T} \xi_{i t}, \\
& \mathbf{v}_{i}^{* \prime} \mathbf{v}_{i}^{*}=\left(1-\hat{\rho}^{2}\right) \psi_{i 1}^{2}+(\rho-\hat{\rho})^{2} \sum_{t=2}^{T}\left(f_{i, t-1}^{2}+2(\rho-\hat{\rho}) \sum_{t=2}^{T} \nu_{i, t-1} e_{i t}+\sum_{t=2}^{T} \xi_{i t}^{2} .\right.
\end{aligned}
$$

Proof. Note that $\mathbf{x}_{i}^{*}=C \mathbf{x}_{i}=\left(\sqrt{1-\hat{\rho}^{2}}, 2-\hat{\rho}, 3-2 \hat{\rho}, \cdots, T-\hat{\rho}(T-1)\right)^{\prime}$ and $\hat{\iota}_{T}^{\alpha}=(\hat{\alpha}, 1,1, \cdots, 1)^{\prime}$ with $\hat{\alpha}=\sqrt{(1+\hat{\rho}) /(1-\hat{\rho})}$. We have

$$
\begin{aligned}
& \mathbf{x}_{i}^{* \prime} \mathbf{x}_{i}^{*}=\left(1-\hat{\rho}^{2}\right)+\sum_{t=2}^{T}\left[(t-\hat{\rho}(t-1)]^{2}=\left(1-\hat{\rho}^{2}\right)+\sum_{t=2}^{T}[(1-\hat{\rho}) t+\hat{\rho}]^{2}\right. \\
& =(1-\hat{\rho})^{2} \sum_{t=2}^{T} t^{2}+2 \hat{\rho}(1-\hat{\rho}) \sum_{t=2}^{T}\left(+\hat{\rho}^{2}(T-1)+\left(1-\hat{\rho}^{2}\right)\right. \\
& \hat{\iota}_{T}^{\alpha \prime} \mathbf{x}_{i}^{*}=(1+\hat{\rho})+\sum_{t=2}^{T}(t-\hat{\rho}(t-1))=(1+\hat{\rho})+\sum_{t=2}^{T}[(1-\hat{\rho}) t+\hat{\rho}] \\
& =(1-\hat{\rho}) \sum_{t=2}^{T}(+\hat{\rho}(T-1)+(1+\hat{\rho}) \text {, } \\
& \mathbf{x}_{i}^{* \prime} \mathbf{v}_{i}^{*}=\left(1-\hat{\rho}^{2}\right) v_{i 1}+\sum_{t=2}^{T}(t-\hat{\rho}(t-1))\left(v_{i t}-\hat{\rho} v_{i, t-1}\right) \\
& =\left(1-\hat{\rho}^{2}\right) \nu_{i 1}+\sum_{t=2}^{T}[(1-\hat{\rho}) t+\hat{\rho}]\left[(\rho-\hat{\rho}) \nu_{i, t-1}+e_{i t}\right] \\
& =\left(1-\hat{\rho}^{2}\right) \psi_{i 1}+(1-\hat{\rho})(\rho-\hat{\rho}) \sum_{t=2}^{T} t \nu_{i, t-1}+\hat{\rho}(\rho-\hat{\rho}) \sum_{t=2}^{T} \nu_{i, t-1}+(1-\hat{\rho}) \sum_{t=2}^{T} t e_{i t}+\hat{\rho} \sum_{t=2}^{T} \oint_{i t} \text {, } \\
& \hat{\iota}_{T}^{\alpha \prime} \mathbf{v}_{i}^{*}=(1+\hat{\rho}) v_{i 1}+\sum_{t=2}^{T}\left(v_{i t}-\hat{\rho} v_{i, t-1}\right)=(1+\hat{\rho}) v_{i 1}+\sum_{t=2}^{T}\left[(\rho-\hat{\rho}) \nu_{i, t-1}+e_{i t}\right] \\
& =(1+\hat{\rho}) v_{i 1}+(\rho-\hat{\rho}) \sum_{t=2}^{T} \nu_{i, t-1}+\sum_{t=2}^{T} \oint_{i t}
\end{aligned}
$$


and

$$
\begin{aligned}
\mathbf{v}_{i}^{* \prime} \mathbf{v}_{i}^{*} & =\left(1-\hat{\rho}^{2}\right) v_{i 1}^{2}+\sum_{t=2}^{T}\left(v_{i t}-\hat{\rho} v_{i, t-1}\right)^{2}=\left(1-\hat{\rho}^{2}\right) \nu_{i 1}^{2}+\sum_{t=2}^{T}\left[(\rho-\hat{\rho}) \nu_{i, t-1}+e_{i t}\right]^{2} \\
& =\left(1-\hat{\rho}^{2}\right) \nu_{i 1}^{2}+(\rho-\hat{\rho})^{2} \sum_{t=2}^{T}\left(_{i, t-1}^{2}+2(\rho-\hat{\rho}) \sum_{t=2}^{T} \nu_{i, t-1} e_{i t}+\sum_{t=2}^{T} \oint_{i t}^{2} .\right.
\end{aligned}
$$

\section{Proof of Theorem 3}

Proof. Consider part (1). For a fixed $n$, from Lemma 1, we have

$$
\begin{aligned}
& \left.\left.\frac{1}{T^{3}} \mathbf{x}_{i}^{* \prime} \mathbf{x}_{i}^{*}=(1-\hat{\rho})^{2} \quad \frac{1}{T^{3}} \sum_{t=1}^{T} t^{2}\right)+\frac{2}{T} \hat{\rho}(1-\hat{\rho}) \quad \frac{1}{T^{2}} \sum_{t=1}^{T} t\right)( \\
& +\frac{1}{T^{2}}\left(\frac{\not}{T}-1\right)\left(\hat{\rho}^{2}+\frac{1}{T^{3}}\left(1-\hat{\rho}^{2}\right) p \frac{(1-\rho)^{2}}{3},\right. \\
& \left.\frac{1}{T^{2}} \hat{\iota}_{T}^{\alpha \prime} \mathbf{x}_{i}^{*}=(1-\hat{\rho}) \quad \frac{1}{T^{2}} \sum_{t=1}^{T} t\right)\left(+\frac{1}{T}\left(\frac{T-1}{T}\right) \hat{\rho}+\frac{1}{T^{2}}(1+\hat{\rho}) \stackrel{p}{\rightarrow} \frac{1-\rho}{2},\right. \\
& \left.\left.\frac{1}{T^{3 / 2}} \mathbf{x}_{i}^{* \prime} \mathbf{v}_{i}^{*}=\frac{1}{T^{3 / 2}}\left(1-\hat{\rho}^{2}\right) \psi_{i 1}+(1-\hat{\rho})(\rho-\hat{\rho}) \frac{1}{T^{3 / 2}} \sum_{t=1}^{T} t \nu_{i, t-1}\right)+\frac{1}{\sqrt{T}} \hat{\rho}(\rho-\hat{\rho}) \frac{1}{\sqrt{T}} \sum_{t=1}^{T} \nu_{i, t-1}\right)( \\
& \left.\left.+(1-\hat{\rho}) \frac{1}{T^{3 / 2}} \sum_{t=1}^{T} t e_{i t}\right)+\frac{1}{T} \hat{\rho} \quad \frac{1}{\sqrt{T}} \sum_{t=1}^{T} e_{i t}\right)\left(\stackrel{d}{\rightarrow}(1-\rho) \sigma_{e} \int r d W_{i},\right. \\
& \left.\frac{1}{\sqrt{T}} \hat{\iota}_{T}^{\alpha \prime} \mathbf{v}_{i}^{*}=\frac{1}{\sqrt{T}}(1+\hat{\rho}) \nu_{i 1}+(\rho-\hat{\rho}) \quad \frac{1}{\sqrt{T}} \sum_{t=1}^{T} \nu_{i, t-1}\right)\left(+\frac{1}{\sqrt{T}} \sum_{t=1}^{T} e_{i t} \stackrel{d}{\rightarrow} \sigma_{e} W_{i}(1),\right. \\
& \frac{1}{T} \mathbf{v}_{i}^{* \prime} \mathbf{v}_{i}^{*}=\left(1-\hat{\rho}^{2}\right)\left(\frac{1}{T} \nu_{i 1}^{2}+(\rho-\hat{\rho})^{2} \frac{1}{T} \sum_{t=2}^{T} \nu_{i, t-1}^{2}+(\rho-\hat{\rho}) \frac{2}{T} \sum_{t=2}^{T} \nu_{i, t-1} e_{i t}+\frac{1}{T} \sum_{t=2}^{T} e_{i t}^{2} \stackrel{d}{\rightarrow} \sigma_{e}^{2},\right.
\end{aligned}
$$

and

$$
\frac{1}{T} \hat{d}^{2}=\frac{T-1}{T}+\frac{1+\hat{\rho}}{T(1-\hat{\rho})} \stackrel{p}{\rightarrow} 1
$$

as $T \rightarrow \infty$, using $\frac{1}{T^{3}} \sum_{t=1}^{T} t^{2} \stackrel{p}{\rightarrow} \frac{1}{3}, \frac{1}{T^{2}} \sum_{t=1}^{T} t \stackrel{p}{\rightarrow} \frac{1}{2}, \frac{1}{T^{3 / 2}} \sum_{t=1}^{T} t \nu_{i, t-1} \stackrel{d}{\rightarrow} \sigma_{v} \int r d W_{i}, \frac{1}{\sqrt{T}} \sum_{t=1}^{T} \nu_{i, t-1} \stackrel{d}{\rightarrow}$ $\sigma_{v} W_{i}(1), \frac{1}{T^{3 / 2}} \sum_{t=1}^{T} t e_{i t} \stackrel{d}{\rightarrow} \sigma_{e} \int r d W_{i}, \frac{1}{\sqrt{T}} \sum_{t=1}^{T} e_{i t} \stackrel{d}{\rightarrow} \sigma_{e} W_{i}(1)$ and $\hat{\rho} \stackrel{p}{\rightarrow} \rho$. Therefore, for a fixed $n$, we have

$$
\frac{1}{T^{3}} \hat{F}_{1}=\frac{1}{T^{3}} \mathbf{x}_{i}^{* \prime} \mathbf{x}_{i}^{*}-\frac{T}{\hat{d}^{2}}\left(\frac{1}{T^{2}} \hat{\boldsymbol{\iota}}_{T}^{\alpha \prime} \mathbf{x}_{i}^{*}\right)^{2} \stackrel{p}{\rightarrow} \frac{(1-\rho)^{2}}{3}-\left(\frac{1-\rho}{2}\right)^{2}=\frac{(1-\rho)^{2}}{12}
$$


and

$$
\begin{aligned}
\frac{\sqrt{n}}{T^{3 / 2}} \hat{F}_{2}= & \frac{1}{\sqrt{n} T^{3 / 2}} \sum_{i=1}^{n}\left(x_{i}^{* \prime} \mathbf{v}_{i}^{*}-\frac{\frac{1}{T^{2}} \hat{\boldsymbol{\iota}}_{T}^{\alpha \prime} \mathbf{x}_{i}^{*}}{\frac{1}{T} \hat{d}^{2}} \frac{1}{\sqrt{n T}} \sum_{i=1}^{n} \hat{\boldsymbol{\iota}}_{T}^{\alpha \prime} \mathbf{v}_{i}^{*}\right) \\
& \stackrel{d}{\rightarrow} \frac{1}{\sqrt{n}} \sum_{i=1}^{n}\left[(1-\rho) \sigma_{e} \int\left(r d W_{i}\right]-\left(\frac{1-\rho}{2}\right)\left(\frac{1}{\sqrt{n}} \sum_{i=1}^{n}\left[\sigma_{e} W_{i}(1)\right]\right.\right. \\
= & (1-\rho) \sigma_{e} \frac{1}{\sqrt{n}} \sum_{i=1}^{n}\left[\int\left(r d W_{i}-\frac{1}{2} W_{i}(1)\right] .\right.
\end{aligned}
$$

Since $\int\left(r d W_{i}-\frac{1}{2} W_{i}(1) \sim N\left(0, \frac{1}{12}\right)\right.$, we have $\frac{\sqrt{n}}{T^{3 / 2}} \hat{F}_{2} \stackrel{d}{\rightarrow} N\left(0, \frac{(1-\rho)^{2} \sigma_{e}^{2}}{12}\right)$ as $(n, T) \rightarrow \infty$. It is easy to show that $\hat{\sigma}_{e}^{2}$ is consistent for $\sigma_{e}^{2}$. Finally, we have

$$
t_{F E-F G L S}=\frac{\frac{\sqrt{n}}{T^{3 / 2}} \hat{F}_{2}}{\sqrt{\hat{\sigma}_{e}^{2} \frac{1}{T^{3}} \hat{F}_{1}}} \stackrel{d}{\rightarrow} \frac{N\left(0, \frac{(1-\rho)^{2} \sigma_{e}^{2}}{12}\right)}{\frac{(1-\rho)^{2} \sigma_{e}^{2}}{12}}(=N(0,1)
$$

as $(n, T) \rightarrow \infty$.

Consider part (2). For a fixed $n$, from Lemma 1, we have

$$
\begin{aligned}
& \left.\left.\frac{1}{T} \mathbf{x}_{i}^{* \prime} \mathbf{x}_{i}^{*}=[T(1-\hat{\rho})]^{2} \frac{1}{T^{3}} \sum_{t=2}^{T} t^{2}\right)+2 \hat{\rho}[T(1-\hat{\rho})] \frac{1}{T^{2}} \sum_{t=2}^{T} t\right)( \\
& +\hat{\rho}^{2}\left(\frac{T-1}{T}\right)\left(+\frac{1}{T^{2}}\left[T\left(1-\hat{\rho}^{2}\right)\right] \stackrel{p}{\longrightarrow} \frac{1}{3} \kappa^{2}-\kappa+1,\right. \\
& \left.\frac{1}{T} \hat{\iota}_{T}^{\alpha \prime} \mathbf{x}_{i}^{*}=[T(1-\hat{\rho})] \quad \frac{1}{T^{2}} \sum_{t=1}^{T} t\right)\left(+\hat{\rho}\left(\frac{T-1}{T}\right)+\frac{1}{T}(1+\hat{\rho}) \stackrel{p}{\rightarrow}-\frac{1}{2} \kappa+1,\right.
\end{aligned}
$$

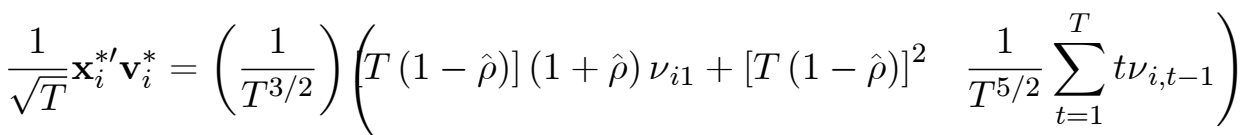

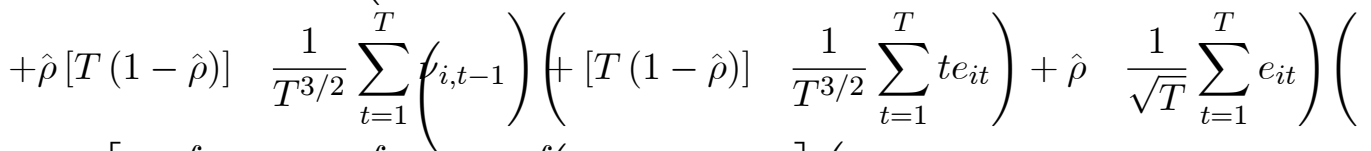

$$
\begin{aligned}
& \stackrel{d}{\rightarrow} \sigma_{e}\left[\kappa^{2} \int r W_{i}-\kappa \int W_{i}-\kappa \int\left(r d W_{i}+W_{i}(1)\right](\right. \\
& \left.\frac{\mathbf{1}}{\sqrt{T}} \hat{\iota}_{T}^{\alpha \prime} \mathbf{v}_{i}^{*}=\frac{1}{\sqrt{T}}(1+\hat{\rho}) \nu_{i 1}+[T(1-\hat{\rho})] \quad \frac{1}{T^{3 / 2}} \sum_{t=1}^{T} \nu_{i, t-1}\right)\left(+\frac{1}{\sqrt{T}} \sum_{t=1}^{T} e_{i t} \stackrel{d}{\rightarrow} \sigma_{e}\left[-\kappa \int\left(W_{i}+W_{i}(1)\right],\right.\right. \\
& \frac{1}{T} \mathbf{v}_{i}^{* \prime} \mathbf{v}_{i}^{*}=\frac{1+\hat{\rho}}{T}[T(1-\hat{\rho})] \frac{1}{T} \nu_{i 1}^{2}+\frac{1}{T^{3 / 2}}[T(1-\hat{\rho})]^{2} \frac{1}{T^{3 / 2}} \sum_{t=2}^{T} \nu_{i, t-1}^{2} \\
& +\frac{1}{T}[T(1-\hat{\rho})] \frac{2}{T} \sum_{t=2}^{T} \nu_{i, t-1} e_{i t}+\frac{1}{T} \sum_{t=2}^{T} e_{i t}^{2} \stackrel{d}{\rightarrow} \sigma_{e}^{2}
\end{aligned}
$$


and

$$
\frac{T}{\hat{d}^{2}}=\frac{T}{\frac{1+\widehat{\rho}}{1-\widehat{\rho}}+T-1}=\frac{T(1-\widehat{\rho})}{2 \widehat{\rho}+T(1-\widehat{\rho})} \stackrel{p}{\rightarrow} \frac{-\kappa}{2-\kappa}
$$

as $T \rightarrow \infty$, using $\frac{1}{T^{3}} \sum_{t=1}^{T} t^{2} \stackrel{p}{\rightarrow} \frac{1}{3}, \frac{1}{T^{2}} \sum_{t=1}^{T} t \stackrel{p}{\rightarrow} \frac{1}{2}, \frac{1}{T^{5 / 2}} \sum_{t=1}^{T} t \nu_{i, t-1} \stackrel{d}{\rightarrow} \int r W_{i}, \frac{1}{T^{3 / 2}} \sum_{t=1}^{T} \nu_{i, t-1} \stackrel{d}{\rightarrow}$ $\int W_{i}, \frac{1}{T^{3 / 2}} \sum_{t=1}^{T} t e_{i t} \stackrel{d}{\rightarrow} \int r d W_{i}, \frac{1}{\sqrt{T}} \sum_{t=1}^{T} e_{i t} \stackrel{d}{\rightarrow} W_{i}(1)$ and $T(1-\hat{\rho}) \stackrel{p}{\rightarrow}-\kappa$. Therefore, for a fixed $n$, we have

$$
\frac{1}{T} \hat{F}_{1}=\frac{1}{T} \mathbf{x}_{i}^{* \prime} \mathbf{x}_{i}^{*}-\frac{[T(1-\hat{\rho})]\left(\frac{1}{T} \hat{\boldsymbol{\iota}}_{T}^{\alpha \prime} \mathbf{x}_{i}^{*}\right)^{2}}{(1-\hat{\rho}) \hat{d}^{2}} \stackrel{p}{\rightarrow} \frac{1}{3} \kappa^{2}-\kappa+1-\frac{-\kappa\left(-\frac{1}{2} \kappa+1\right)^{2}}{2-\kappa}=\frac{-\kappa^{3}+8 \kappa^{2}-24 \kappa+24}{12(2-\kappa)},
$$

and

$$
\begin{aligned}
\sqrt{\frac{h}{f}} \hat{F}_{2}= & \frac{1}{\sqrt{n T}} \sum_{i=1}^{n} \boldsymbol{x}_{i}^{* \prime} \mathbf{v}_{i}^{*}-\frac{[T(1-\hat{\rho})]\left(\frac{1}{T} \hat{\boldsymbol{\iota}}_{T}^{\alpha \prime} \mathbf{x}_{i}^{*}\right)}{(1-\hat{\rho}) \hat{d}^{2}}\left(\frac{1}{\sqrt{n T}} \sum_{i=1}^{n} \boldsymbol{f}_{T}^{\alpha \prime} \mathbf{v}_{i}^{*}\right)( \\
& \stackrel{d}{\rightarrow} \frac{1}{\sqrt{n}} \sum_{i=1}^{n} \sigma_{e}\left[\kappa^{2} \int r W_{i}-\kappa \int W_{i}-\kappa \int\left(r d W_{i}+W_{i}(1)\right]\right. \\
& -\frac{-\kappa\left(-\frac{1}{2} \kappa+1\right)}{2-\kappa}\left(\frac { 1 } { \sqrt { n } } \sum _ { i = 1 } ^ { n } \sigma _ { e } \left[-\kappa \int\left(W_{i}+W_{i}(1)\right](\right.\right. \\
= & \frac{\sigma_{e}}{\sqrt{n}} \sum_{i=1}^{n}\left[\kappa^{2} \int r W_{i}-\kappa \int W_{i}-\kappa \int\left(r d W_{i}+W_{i}(1)+\frac{\kappa}{2}\left(-\kappa \int\left(W_{i}+W_{i}(1)\right)\right]\right.\right.
\end{aligned}
$$

as $T \rightarrow \infty$. Since $\int\left(r d W_{i}=W_{i}(1)-\int\left(W_{i}, W_{i}(1)=\int d W_{i}, \int r W_{i}=\frac{1}{2} \int W_{i} d r^{2}=\frac{1}{2}\left[W_{i}(1)-\int r^{2} d W_{i}\right]=\right.\right.$ $\frac{1}{2} \int\left(1-r^{2}\right) d W_{i}$, we know that

$$
\begin{aligned}
& \kappa^{2} \int r W_{i}-\kappa \int W_{i}-\kappa \int\left(r d W_{i}+W_{i}(1)+\frac{\kappa}{2}\left(-\kappa \int W_{i}+W_{i}(1)\right)(\right. \\
= & \kappa^{2} \int\left(r W_{i}+(1-\kappa) W_{i}(1)+\frac{\kappa}{2}\left(-\kappa \int W_{i}+W_{i}(1)\right)(\right. \\
= & \int\left[( \frac { 1 } { 2 } \kappa ^ { 2 } r ^ { 2 } + \frac { 1 } { 2 } \kappa ^ { 2 } r + ( 1 - \frac { 1 } { 2 } \kappa ) ] \left(W_{i},\right.\right.
\end{aligned}
$$

which follows a normal distribution with zero mean and variance $\int\left[f_{2}^{\frac{1}{2}} \kappa^{2} r^{2}+\frac{1}{2} \kappa^{2} r+\left(1-\frac{1}{2} \kappa\right)\right]^{2} d r=$ $\frac{1}{120} \kappa^{4}-\frac{1}{12} \kappa^{3}+\frac{5}{12} \kappa^{2}-\kappa+1$. Hence

$$
\sqrt{\frac{n}{T}} \hat{F}_{2} \stackrel{d}{\rightarrow} \sigma_{e} N\left(\oint, \frac{1}{120} \kappa^{4}-\frac{1}{12} \kappa^{3}+\frac{5}{12} \kappa^{2}-\kappa+1\right)(
$$

as $(n, T) \rightarrow \infty$. It is easy to show that $\hat{\sigma}_{e}^{2}$ is consistent for $\sigma_{e}^{2}$. Finally, we have

$$
\begin{aligned}
t_{F E-F G L S} & =\frac{\sqrt{\frac{n}{T}} \hat{F}_{2}}{\sqrt{\sigma_{e}^{2} \frac{1}{T} \hat{F}_{1}}} \stackrel{d}{\rightarrow} \frac{\sigma_{e} N\left(\emptyset, \frac{1}{120} \kappa^{4}-\frac{1}{12} \kappa^{3}+\frac{5}{12} \kappa^{2}-\kappa+1\right)}{\sigma_{e} \sqrt{\frac{\kappa^{4}-9 \kappa^{3}+33 \kappa^{2}-54 \kappa+36}{12\left(\kappa^{2}-3 \kappa+3\right)}}}( \\
& \left.=N \quad 0, \frac{\left(\kappa^{2}-3 \kappa+3\right)\left(\kappa^{4}-10 \kappa^{3}+50 \kappa^{2}-120 \kappa+120\right)}{10\left(\kappa^{4}-\left(9 \kappa^{3}+33 \kappa^{2}-54 \kappa+36\right)\right.}\right)
\end{aligned}
$$


as $(n, T) \rightarrow \infty$.

\section{Lemma 2}

and

$$
\begin{aligned}
\sum_{i=1}^{n} \sum_{t=2}^{T} \hat{\nu}_{i, t-1}^{2}= & \sum_{i=1}^{n} \sum_{t=2}^{T}\left(v_{i, t-1}-\bar{v}_{i .}\right)^{2}+\left(\hat{\beta}_{F E}-\beta\right)^{2} \sum_{i=1}^{n} \sum_{t=2}^{T}(t-1-\bar{t})^{2} \\
& -2\left(\hat{\beta}_{F E}-\beta\right) \sum_{i=1}^{n} \sum_{t=2}^{T}\left(v_{i, t-1}-\bar{v}_{i .}\right)(t-1-\bar{t})
\end{aligned}
$$

$$
\begin{aligned}
& \sum_{i=1}^{n} \sum_{t=2}^{T}\left(\hat{\nu}_{i t}-\rho \hat{\nu}_{i, t-1}\right) \hat{\nu}_{i, t-1} \\
= & {\left[\sum_{i=1}^{n} \sum_{t=2}^{T} \xi_{i t}\left(v_{i, t-1}-\bar{v}_{i .}\right)+(1-\rho) \sum_{i=1}^{n} \hat{f}_{i .}\left(v_{i T}-\bar{v}_{i .}\right)\right](} \\
& -\rho\left(\hat{\beta}_{F E}-\beta\right)\left[(1-\rho) \sum_{i=1}^{n} \sum_{t=2}^{T}\left(v_{i, t-1}-\bar{v}_{i .}\right)(t-\bar{t})-(2 \rho-1) \sum_{i=1}^{n}\left(v_{i T}-\bar{v}_{i .}\right)\right]( \\
& -\left(\hat{\beta}_{F E}-\beta\right)\left[\sum_{i=1}^{n} \sum_{t=2}^{T} \oint_{i t}(t-1-\bar{t})+\frac{1}{2}(T-1)(1-\rho) \sum_{i=1}^{n} \hat{f}_{i .}\right]\left(\hat{\beta}_{F E}-\beta\right)^{2}\left[n(1-\rho) \sum_{t=2}^{T}(t-1-\bar{t})^{2}+\frac{1}{2} n(T-1)\right](
\end{aligned}
$$

When $\rho=1$, it reduces to

$$
\begin{aligned}
\sum_{i=1}^{n} \sum_{t=2}^{T} \Delta \hat{\nu}_{i t} \hat{\nu}_{i, t-1}= & \sum_{i=1}^{n} \sum_{t=2}^{T} \oint_{i t}\left(v_{i, t-1}-\bar{v}_{i .}\right)-\left(\hat{\beta}_{F E}-\beta\right)\left[\sum_{i=1}^{n}\left(v_{i T}-\bar{v}_{i .}\right)\right]( \\
& -\left(\hat{\beta}_{F E}-\beta\right)\left[\sum_{i=1}^{n} \sum_{t=2}^{T} \phi_{i t}(t-1-\bar{t})\right]\left(+\frac{n(T-1)}{2}\left(\hat{\beta}_{F E}-\beta\right)^{2} .\right.
\end{aligned}
$$

Proof. Note that $\hat{\nu}_{i t}=\left(y_{i t}-\bar{y}_{i .}\right)-\hat{\beta}_{F E}(t-\bar{t})=\left(v_{i t}-\bar{v}_{i .}\right)-\left(\hat{\beta}_{F E}-\beta\right)(t-\bar{t})$ and hence

Also, we have

$$
\begin{aligned}
\sum_{i=1}^{n} \sum_{t=2}^{T} \hat{\nu}_{i, t-1}^{2}= & \sum_{i=1}^{n} \sum_{t=2}^{T}\left(v_{i, t-1}-\bar{v}_{i .}\right)^{2}+\left(\hat{\beta}_{F E}-\beta\right)^{2} \sum_{i=1}^{n} \sum_{t=2}^{T}(t-1-\bar{t})^{2} \\
& -2\left(\hat{\beta}_{F E}-\beta\right) \sum_{t=1}^{n} \sum_{t=2}^{T}\left(v_{i, t-1}-\bar{v}_{i .}\right)(t-1-\bar{t}) .
\end{aligned}
$$

$$
\begin{aligned}
& \hat{\nu}_{i t}-\rho \hat{\nu}_{i, t-1} \\
= & {\left[\left(v_{i t}-\bar{v}_{i .}\right)-\left(\hat{\beta}_{F E}-\beta\right)(t-\bar{t})\right]-\rho\left[\left(v_{i, t-1}-\bar{v}_{i .}\right)-\left(\hat{\beta}_{F E}-\beta\right)(t-1-\bar{t})\right](} \\
= & \left.\left.e_{i t}-(1-\rho) \bar{v}_{i .}-\left(\hat{\beta}_{F E}-\beta\right)\right](1-\rho)(t-\bar{t})+\rho\right]
\end{aligned}
$$


and hence

$$
\begin{aligned}
& \sum_{i=1}^{n} \sum_{t=2}^{T}\left(\hat{\nu}_{i t}-\rho \hat{\nu}_{i, t-1}\right) \hat{\nu}_{i, t-1} \\
= & \sum_{i=1}^{n} \sum_{t=2}^{T}\left[\left(f_{i t}-(1-\rho) \bar{v}_{i .}-\left(\hat{\beta}_{F E}-\beta\right)((1-\rho)(t-\bar{t})+\rho)\right]\left[\left(v_{i, t-1}-\bar{v}_{i .}\right)-\left(\hat{\beta}_{F E}-\beta\right)(t-1-\bar{t})\right](\right. \\
= & \sum_{i=1}^{n} \sum_{t=2}^{T}\left(e_{i t}-(1-\rho) \bar{v}_{i .}\right)\left(v_{i, t-1}-\bar{v}_{i .}\right)-\left(\hat{\beta}_{F E}-\beta\right) \sum_{i=1}^{n} \sum_{t=2}^{T}\left(v_{i, t-1}-\bar{v}_{i .}\right)((1-\rho)(t-\bar{t})+\rho) \\
& -\left(\hat{\beta}_{F E}-\beta\right) \sum_{i=1}^{n} \sum_{t=2}^{T}\left(e_{i t}-(1-\rho) \bar{v}_{i .}\right)(t-1-\bar{t})+\left(\hat{\beta}_{F E}-\beta\right)^{2} \sum_{i=1}^{n} \sum_{t=2}^{T}((1-\rho)(t-\bar{t})+\rho)(t-1-\bar{t}) \\
= & I+I I+I I I+I V .
\end{aligned}
$$

It is easy to see that

$$
\begin{aligned}
I & =\sum_{i=1}^{n} \sum_{t=2}^{T}\left(e_{i t}-(1-\rho) \bar{v}_{i .}\right)\left(v_{i, t-1}-\bar{v}_{i .}\right) \\
& =\sum_{i=1}^{n} \sum_{t=2}^{T} \oint_{i t}\left(v_{i, t-1}-\bar{v}_{i .}\right)-(1-\rho) \sum_{i=1}^{n} \sum_{t=2}^{T} f_{i .}\left(v_{i, t-1}-\bar{v}_{i .}\right) \\
& =\sum_{i=1}^{n} \sum_{t=2}^{T} \oint_{i t}\left(v_{i, t-1}-\bar{v}_{i .}\right)+(1-\rho) \sum_{i=1}^{n} f_{i .}\left(v_{i T}-\bar{v}_{i .}\right),
\end{aligned}
$$

using $\sum_{t=2}^{T}\left(v_{i, t-1}-\bar{v}_{i .}\right)=\left[\sum_{t=1}^{T}\left(v_{i t}-\bar{v}_{i .}\right)\right]-\left(v_{i T}-\bar{v}_{i .}\right)=-\left(v_{i T}-\bar{v}_{i .}\right)$. For term $I I$, we have

$$
\begin{aligned}
& \sum_{i=1}^{n} \sum_{t=2}^{T}\left(v_{i, t-1}-\bar{v}_{i .}\right)((1-\rho)(t-\bar{t})+\rho) \\
= & (1-\rho) \sum_{i=1}^{n} \sum_{t=2}^{T}\left(v_{i, t-1}-\bar{v}_{i .}\right)(t-\bar{t})+(2 \rho-1) \sum_{i=1}^{n} \sum_{t=2}^{T}\left(v_{i, t-1}-\bar{v}_{i .}\right) \\
= & (1-\rho) \sum_{i=1}^{n} \sum_{t=2}^{T}\left(v_{i, t-1}-\bar{v}_{i .}\right)(t-\bar{t})-(2 \rho-1) \sum_{i=1}^{n}\left(v_{i T}-\bar{v}_{i .}\right) .
\end{aligned}
$$

For term $I I I$, we have

$$
\begin{aligned}
& \sum_{i=1}^{n} \sum_{t=2}^{T}\left(e_{i t}-(1-\rho) \bar{v}_{i .}\right)(t-1-\bar{t}) \\
= & \sum_{i=1}^{n} \sum_{t=2}^{T} \oint_{i t}(t-1-\bar{t})-(1-\rho) \sum_{i=1}^{n} \bar{v}_{i \cdot} \sum_{t=2}^{T}(t-1-\bar{t}) \\
= & \sum_{i=1}^{n} \sum_{t=2}^{T} \oint_{i t}(t-1-\bar{t})+\frac{1}{2}(T-1)(1-\rho) \sum_{i=1}^{n} \phi_{i .},
\end{aligned}
$$


since $\sum_{t=2}^{T}(t-1-\bar{t})=\left[\sum_{t=1}^{T}(t-\bar{t})\right]-(T-\bar{t})=-(T-\bar{t})=-\frac{T-1}{2}$. For term $I V$, we have

$$
\begin{aligned}
& \sum_{i=1}^{n} \sum_{t=2}^{T}((1-\rho)(t-\bar{t})+\rho)(t-1-\bar{t}) \\
= & n \sum_{t=2}^{T}((1-\rho)(t-1-\bar{t})+1)(t-1-\bar{t}) \\
= & n(1-\rho) \sum_{t=2}^{T}(t-1-\bar{t})^{2}+n \sum_{t=2}^{T}(t-1-\bar{t}) \\
= & n(1-\rho) \sum_{t=2}^{T}(t-1-\bar{t})^{2}+\frac{1}{2} n(T-1) .
\end{aligned}
$$

Therefore,

$$
\begin{aligned}
& \sum_{i=1}^{n} \sum_{t=2}^{T}\left(\hat{\nu}_{i t}-\rho \hat{\nu}_{i, t-1}\right) \hat{\nu}_{i, t-1} \\
= & {\left[\sum_{i=1}^{n} \sum_{t=2}^{T} \oint_{i t}\left(v_{i, t-1}-\bar{v}_{i .}\right)+(1-\rho) \sum_{i=1}^{n} \hat{f}_{i .}\left(v_{i T}-\bar{v}_{i .}\right)\right](} \\
& -\rho\left(\hat{\beta}_{F E}-\beta\right)\left[(1-\rho) \sum_{i=1}^{n} \sum_{t=2}^{T}\left(v_{i, t-1}-\bar{v}_{i .}\right)(t-\bar{t})-(2 \rho-1) \sum_{i=1}^{n}\left(v_{i T}-\bar{v}_{i .}\right)\right]( \\
& -\left(\hat{\beta}_{F E}-\beta\right)\left(\sum_{i=1}^{n} \sum_{t=2}^{T} \oint_{i t}(t-1-\bar{t})+\frac{1}{2}(T-1)(1-\rho) \sum_{i=1}^{n} \hat{t}_{i .}\right]\left(\hat{\beta}_{F E}-\beta\right)^{2}\left[n(1-\rho) \sum_{t=2}^{T}(t-1-\bar{t})^{2}+\frac{1}{2} n(T-1)\right](
\end{aligned}
$$

\section{Proof of Theorem 4}

Proof. Note that

$$
\hat{\rho}-\rho=\frac{\sum_{i=1}^{n} \sum_{t=2}^{T}\left(\hat{\nu}_{i t}-\rho \hat{\nu}_{i, t-1}\right) \hat{\nu}_{i, t-1}}{\sum_{i=1}^{n} \sum_{t=2}^{T} \hat{\nu}_{i, t-1}^{2}} .
$$

Consider (1). First, from Lemma 2, we have

$$
\begin{aligned}
\frac{1}{n T} \sum_{i=1}^{n} \sum_{t=2}^{T} \hat{\nu}_{i, t-1}^{2}= & \frac{1}{n T} \sum_{i=1}^{n} \sum_{t=2}^{T}\left(v_{i, t-1}-\bar{v}_{i .}\right)^{2}+\frac{1}{n T}\left[\sqrt{n} T^{3 / 2}\left(\hat{\beta}_{F E}-\beta\right)\right]^{2}\left[\frac{1}{T^{3}} \sum_{t=2}^{T}(t-1-\bar{t})^{2}\right]( \\
& -\frac{2}{n T}\left[\sqrt{n} T^{3 / 2}\left(\hat{\phi}_{F E}-\beta\right)\right]\left(\frac{1}{\sqrt{n} T^{3 / 2}} \sum_{i=1}^{n} \sum_{t=2}^{T}\left(v_{i, t-1}-\bar{v}_{i .}\right)(t-1-\bar{t})\right](
\end{aligned}
$$


Notice that

$$
\frac{1}{n T} \sum_{i=1}^{n} \sum_{t=2}^{T}\left(v_{i, t-1}-\bar{v}_{i .}\right)^{2} \stackrel{p}{\rightarrow} \frac{\sigma_{e}^{2}}{1-\rho^{2}}
$$

by Lemma 3.1 in Baltagi, Kao and Liu (2008),

$$
\sqrt{n} T^{3 / 2}\left(\hat{\beta}_{F E}-\beta\right) \stackrel{d}{\rightarrow} N\left(0, \frac{12 \sigma_{e}^{2}}{(1-\rho)^{2}}\right)
$$

by Theorem 3 in Kao and Emerson (2004) and

$$
\frac{1}{\sqrt{n} T^{3 / 2}} \sum_{i=1}^{n} \sum_{t=2}^{T}\left(v_{i, t-1}-\bar{v}_{i .}\right)(t-1-\bar{t}) \stackrel{d}{\rightarrow} N\left(\oint, \frac{\sigma_{e}^{2}}{12(1-\rho)^{2}}\right)(
$$

by an equation on page 17 in Kao and Emerson (2004).

Hence we have

$$
\frac{1}{n T} \sum_{i=1}^{n} \sum_{t=2}^{T} \not_{i, t-1}^{2} \stackrel{p}{\rightarrow} \frac{\sigma_{e}^{2}}{1-\rho^{2}}
$$

Also, we have

$$
\begin{aligned}
& \frac{1}{\sqrt{n T}} \sum_{i=1}^{n} \sum_{t=2}^{T}\left(\hat{\nu}_{i t}-\rho \hat{\nu}_{i, t-1}\right) \hat{\nu}_{i, t-1} \\
& =\frac{1}{\sqrt{n T}} \sum_{i=1}^{n} \sum_{t=2}^{T} \xi_{i t}\left(v_{i, t-1}-\bar{v}_{i .}\right)+\frac{1-\rho}{\sqrt{n T}} \sum_{i=1}^{n} \bar{v}_{i .}\left(v_{i T}-\bar{v}_{i .}\right) \\
& -\frac{\rho}{\sqrt{n T}}\left[\sqrt{n} T^{3 / 2}\left(\ddot{\beta}_{F E}-\beta\right)\right]\left[\left(\frac{1-\rho}{\sqrt{n} T^{3 / 2}} \sum_{i=1}^{n} \sum_{t=2}^{T}\left(v_{i, t-1}-\bar{v}_{i .}\right)(t-\bar{t})-\frac{2 \rho-1}{T^{3 / 2}} \frac{1}{\sqrt{n}} \sum_{i=1}^{n}\left(v_{i T}-\bar{v}_{i .}\right)\right)\right]( \\
& -\frac{1}{\sqrt{n T}}\left[\sqrt{n} T^{3 / 2}\left(\ddot{\beta}_{F E}-\beta\right)\right]\left[\left(\frac{1}{\sqrt{n} T^{3 / 2}} \sum_{i=1}^{n} \sum_{t=2}^{T} \xi_{i t}(t-1-\bar{t})+\frac{1}{\sqrt{T}} \frac{(1-\rho)(T-1)}{2 T} \frac{1}{\sqrt{n}} \sum_{i=1}^{n} \bar{v}_{i .}\right)\right]( \\
& +\frac{1}{\sqrt{n T}}\left[\sqrt{n} T^{3 / 2}\left(\tilde{\beta}_{F E}-\beta\right)\right]^{2}\left[(1-\rho)\left[\left(\frac{1}{\mathbb{f}^{3}} \sum_{t=2}^{T}(t-1-\bar{t})^{2}\right]+\frac{1}{T^{2}}\left(\frac{T-1}{2 T}\right)\right]\right. \\
& =I+I I+I I I+I V \text {. }
\end{aligned}
$$

Consider $I$ : Notice that

$$
\begin{aligned}
\frac{1}{\sqrt{n T}} \sum_{i=1}^{n} \sum_{t=2}^{T} \oint_{i t}\left(v_{i, t-1}-\bar{v}_{i .}\right)+\sqrt{\frac{n}{T}} \frac{\sigma_{e}^{2}}{1-\rho}= & \left.\frac{1}{\sqrt{n T}} \sum_{i=1}^{n} \sum_{t=2}^{T} e_{i t} v_{i, t-1}-\sqrt{\frac{n}{T}} \frac{1}{n} \sum_{i=1}^{n} \sum_{t=2}^{T} e_{i t} \bar{v}_{i .}-\frac{\sigma_{e}^{2}}{1-\rho}\right) \\
& \stackrel{d}{\rightarrow} \sigma_{e}^{2} N\left(0, \frac{1}{1-\rho^{2}}\right)(
\end{aligned}
$$

using

$$
\frac{1}{\sqrt{n T}} \sum_{i=1}^{n} \sum_{t=2}^{T} e_{i t} v_{i, t-1} \stackrel{d}{\rightarrow} \sigma_{e}^{2} N\left(0, \frac{1}{1-\rho^{2}}\right)
$$


and

$$
\left.\left.\sqrt{n} \frac{1}{n} \sum_{i=1}^{n} \sum_{t=2}^{T} \xi_{i t} \bar{v}_{i .}-\frac{\sigma_{e}^{2}}{1-\rho}\right)\left(\begin{array}{l}
d \\
\rightarrow
\end{array} \quad 0, \lim _{(n, T) \rightarrow \infty} \operatorname{Var} \sum_{t=2}^{T} \xi_{i t} \bar{v}_{i .}\right)\right)(
$$

by the Lindeberg-Feller central limit theorem and

$$
\left.E \quad \sum_{t=2}^{T} \oint_{i t} \bar{v}_{i .}\right)\left(=E \quad \frac{1}{T} \sum_{t=1}^{T} \sum_{s=2}^{T} \wp_{i t} e_{i s}\right)\left(=\frac{1}{T} \sum_{t=1}^{T} \sum_{s=2}^{t} \notin\left(v_{i t} e_{i s}\right)=\frac{1}{T} \sum_{t=1}^{T} \sum_{j=0}^{t-1} \rho^{j} \sigma_{e}^{2}=\frac{\sigma_{e}^{2}}{1-\rho} .\right.
$$

Together with the fact that

$$
\frac{1}{\sqrt{n T}} \sum_{i=1}^{n} f_{i .}\left(v_{i T}-\bar{v}_{i .}\right)=\frac{1}{\sqrt{n T}} \sum_{i=1}^{n} f_{i .} v_{i T}-\frac{1}{\sqrt{n T}} \sum_{i=1}^{n} f_{i .}^{2} \stackrel{p}{\rightarrow} 0
$$

we have

$$
I+\sqrt{\frac{h}{f}} \frac{\sigma_{e}^{2}}{1-\rho} \stackrel{d}{\rightarrow} \sigma_{e}^{2} N\left(\oint, \frac{1}{1-\rho^{2}}\right)(
$$

Consider II: By Theorem 3 in Kao and Emerson (2000), we have

$$
\sqrt{n} T^{3 / 2}\left(\ddot{\phi}_{F E}-\beta\right) \stackrel{d}{\rightarrow} N\left(\phi, \frac{12 \sigma_{e}^{2}}{(1-\rho)^{2}}\right)(
$$

Also it is easy to show that

$$
\frac{1}{\sqrt{n} T^{3 / 2}} \sum_{i=1}^{n} \sum_{t=2}^{T}\left(v_{i, t-1}-\bar{v}_{i .}\right)(t-\bar{t}) \stackrel{d}{\rightarrow} N\left(\oint, \frac{\sigma_{e}^{2}}{12(1-\rho)^{2}}\right)(
$$

and

Hence the term $I I=o_{p}(1)$.

$$
\frac{1}{\sqrt{n}} \sum_{i=1}^{n}\left(v_{i T}-\bar{v}_{i .}\right)=O_{p}(1)
$$

Consider III: By an equation on page 17 in Kao and Emerson (2004), we have

$$
\frac{1}{\sqrt{n} T^{3 / 2}} \sum_{i=1}^{n} \sum_{t=2}^{T} \oint_{i t}(t-1-\bar{t}) \stackrel{d}{\rightarrow} N\left(\phi, \frac{\sigma_{e}^{2}}{12}\right)(
$$

Also it is easy to show that

Hence the term $I I I=o_{p}(1)$.

$$
\frac{1}{\sqrt{n}} \sum_{i=1}^{n} f_{i .}=O_{p}(1)
$$

Consider $I V$ : It is easy to show that

$$
\frac{1}{n T^{3}} \sum_{i=1}^{n} \sum_{t=2}^{T}(t-\bar{t})^{2} \stackrel{p}{\rightarrow} \frac{1}{12}
$$


and hence the term $I V=o_{p}(1)$.

Hence we have

$$
\frac{1}{\sqrt{n T}} \sum_{i=1}^{n} \sum_{t=2}^{T}\left(\hat{\nu}_{i t}-\rho \hat{\nu}_{i, t-1}\right) \hat{\nu}_{i, t-1}+\sqrt{\frac{n}{T}} \frac{\sigma_{e}^{2}}{1-\rho} \stackrel{d}{\rightarrow} \sigma_{e}^{2} N\left(0, \frac{1}{1-\rho^{2}}\right) .
$$

Therefore,

$$
\begin{aligned}
\sqrt{n T}\left(\hat{\rho}-\rho+\frac{1+\rho}{T}\right)(= & \frac{\frac{1}{\sqrt{n T}} \sum_{i=1}^{n} \sum_{t=2}^{T}\left(\hat{\nu}_{i t}-\rho \hat{\nu}_{i, t-1}\right) \hat{\nu}_{i, t-1}+\sqrt{\frac{n}{T}} \frac{\sigma_{e}^{2}}{1-\rho}}{\frac{1}{n T} \sum_{i}^{n} \sum_{1} \sum_{t=2}^{T} \hat{\nu}_{i, t-1}^{2}}+o_{p}(1) \\
& \stackrel{d}{\rightarrow} \frac{\sigma_{e}^{2} N\left(0, \frac{1}{1-\rho^{2}}\right)}{\frac{\sigma_{e}^{2}}{1-\rho^{2}}}\left(+o_{p}(1)=N\left(0,1-\rho^{2}\right)(\right.
\end{aligned}
$$

as $(n, T) \rightarrow \infty$.

Next we show part (2). First, from Lemma 2, we have

$$
\begin{aligned}
& \frac{1}{n T^{2}} \sum_{i=1}^{n} \sum_{t=2}^{T} \hat{\nu}_{i, t-1}^{2} \\
= & \frac{1}{n T^{2}} \sum_{i=1}^{n} \sum_{t=2}^{T}\left(v_{i, t-1}-\bar{v}_{i .}\right)^{2}-\frac{2}{n}\left[\sqrt{n T}\left(\hat{\beta}_{F E}-\beta\right)\right]\left(\frac{1}{\sqrt{n} T^{5 / 2}} \sum_{i=1}^{n} \sum_{t=2}^{T}\left[(t-1-\bar{t})\left(v_{i t}-\bar{v}_{i .}\right)\right]\right. \\
& +\frac{1}{n}\left[n T\left(\hat{\beta}_{F E}-\beta\right)^{2}\right]\left(\frac{1}{T^{3}} \sum_{t=2}^{T}(t-1-\bar{t})^{2} .\right.
\end{aligned}
$$

Notice that

$$
\sqrt{n T}\left(\hat{\beta}_{F E}-\beta\right) \stackrel{d}{\rightarrow} N\left(0, \frac{6}{5} \sigma_{e}^{2}\right)
$$

by Theorem 4 in Kao and Emerson (2004),

$\frac{1}{\sqrt{n} T^{5 / 2}} \sum_{i=1}^{n} \sum_{t=2}^{T}\left[(t-1-\bar{t})\left(v_{i, t-1}-\bar{v}_{i .}\right)\right]=\frac{1}{\sqrt{n} T^{5 / 2}} \sum_{i=1}^{n} \sum_{t=2}^{T}\left[(t-1-\bar{t}) v_{i, t-1}\right] \stackrel{d}{\rightarrow} N\left(0, \frac{\sigma_{e}^{2}}{120}\right)=O_{p}(1)$

by an equation on page 23 in Kao and Emerson (2004), and

$$
\frac{1}{n T^{3}} \sum_{i=1}^{n} \sum_{t=2}^{T}(t-1-\bar{t})^{2} \rightarrow \frac{1}{12}
$$

Hence we have

$$
\frac{1}{n T^{2}} \sum_{i=1}^{n} \sum_{t=2}^{T} \not_{i, t-1}^{2}=\frac{1}{n T^{2}} \sum_{i=1}^{n} \sum_{t=2}^{T}\left(v_{i, t-1}-\bar{v}_{i .}\right)^{2}+o_{p}(1)
$$


Next, from Lemma 2, we have

$$
\begin{aligned}
& \frac{1}{\sqrt{n} T} \sum_{i=1}^{n} \sum_{t=2}^{T} \Delta \hat{\nu}_{i t} \hat{\nu}_{i, t-1} \\
= & \frac{1}{\sqrt{n} T} \sum_{i=1}^{n} \sum_{t=2}^{T} \phi_{i t}\left(v_{i, t-1}-\bar{v}_{i .}\right)-\frac{1}{\sqrt{n} T}\left[\sqrt{n T}\left(\hat{\beta}_{F E}-\beta\right)\right]\left[\frac{1}{\sqrt{n}} \sum_{i=1}^{n} \frac{1}{\sqrt{T}}\left(v_{i T}-\bar{v}_{i .}\right)\right]( \\
& -\frac{1}{\sqrt{n}}\left[\sqrt{n T}\left(\hat{\phi}_{F E}-\beta\right)\right]\left(\frac{1}{\sqrt{n} T^{3 / 2}} \sum_{i=1}^{n} \sum_{t=2}^{T} e_{i t}(t-1-\bar{t})\right]\left(+\frac{1}{\sqrt{n} T} \frac{T-1}{2 T}\left[\sqrt{n T}\left(\hat{\phi}_{F E}-\beta\right)\right]^{2} .\right.
\end{aligned}
$$

Notice that

$$
\frac{1}{n T} \sum_{i=1}^{n} \sum_{t=2}^{T} \oint_{i t}\left(v_{i, t-1}-\bar{v}_{i .}\right) \stackrel{p}{\rightarrow}-\frac{\sigma_{e}^{2}}{2}
$$

by equation (C5) in Kao (1999),

$$
\begin{gathered}
\frac{1}{\sqrt{n}} \sum_{i=1}^{n}\left(\frac{1}{\sqrt{T}}\left(v_{i T}-\bar{v}_{i .}\right)=\frac{1}{\sqrt{n}} \sum_{i=1}^{n}\left(\frac{v_{i T}}{\sqrt{T}}\right)\left(-\frac{1}{\sqrt{n}} \sum_{i=1}^{n}\left(\frac{1}{T^{3 / 2}} \sum_{t=2}^{T} \wp_{i t}\right)(\stackrel{p}{\rightarrow} 0\right.\right. \\
\frac{1}{\sqrt{n} T^{3 / 2}} \sum_{i=1}^{n} \sum_{t=2}^{T} \xi_{i t}(t-1-\bar{t}) \stackrel{d}{\rightarrow} N\left(\phi, \frac{\sigma_{e}^{2}}{12}\right)
\end{gathered}
$$

by an equation on page 17 in Kao and Emerson (2004), and

$$
\sqrt{n T}\left(\hat{\phi}_{F E}-\beta\right) \stackrel{d}{\rightarrow} N\left(\phi, \frac{6}{5} \sigma_{e}^{2}\right)
$$

by Theorem 4 in Kao and Emerson (2004).

Hence we have

$$
\frac{1}{\sqrt{n} T} \sum_{i=1}^{n} \sum_{t=2}^{T} \hat{A} \hat{\nu}_{i t} \hat{\nu}_{i, t-1}=\frac{1}{\sqrt{n} T} \sum_{i=1}^{n} \sum_{t=2}^{T} \oint_{i t}\left(v_{i, t-1}-\bar{v}_{i .}\right)+o_{p}(1) .
$$

Therefore,

$$
\begin{aligned}
& \sqrt{n} T(\hat{\rho}-1)=\frac{\frac{1}{\sqrt{n} T} \sum_{i=1}^{n} \sum_{t=2}^{T} \Delta \hat{\nu}_{i t} \hat{\nu}_{i, t-1}}{\frac{1}{n T^{2}} \sum_{i}^{n}\left(=1 \sum_{t=2}^{T} \hat{\nu}_{i, t-1}^{2}\right.}=\frac{\frac{1}{\sqrt{n} T} \sum_{i=1}^{n} \sum_{t=2}^{T} e_{i t}\left(v_{i, t-1}-\bar{v}_{i .}\right)}{\frac{1}{n T^{2}} \sum_{i}^{n}\left({ }_{1} \sum_{t=2}^{T}\left(v_{i, t-1}-\bar{v}_{i .}\right)^{2}\right.}+o_{p}(1) \\
& T \rightarrow \rightarrow \infty \text {. As shown in equation (C5) in Kao }(1999),
\end{aligned}
$$

$$
\frac{1}{T} \sum_{t=2}^{T} e_{i t}\left(v_{i, t-1}-\bar{v}_{i .}\right) \stackrel{d}{\rightarrow} \zeta_{3 i}
$$

with $E\left(\zeta_{3 i}\right)=-\frac{\sigma_{e}^{2}}{2}$ and $\operatorname{Var}\left(\zeta_{3 i}\right)=\frac{\sigma_{e}^{4}}{12}$. Also, as shown in equation (C2) in Kao (1999),

$$
\frac{1}{T^{2}} \sum_{t=2}^{T}\left(v_{i, t-1}-\bar{v}_{i .}\right)^{2} \stackrel{d}{\rightarrow} \zeta_{4 i}
$$


with $E\left(\zeta_{4 i}\right)=\frac{\sigma_{e}^{2}}{6}$ and $\operatorname{Var}\left(\zeta_{4 i}\right)=\frac{\sigma_{e}^{4}}{45}$. By equation $(\mathrm{C} 10)$ in Kao (1999), we know

$$
\sqrt{n} T(\hat{\rho}-1)-\sqrt{n} \frac{-\frac{\sigma_{e}^{2}}{2}}{\frac{\sigma_{e}^{2}}{6}} \stackrel{d}{\rightarrow} N\left(\oint, \frac{\frac{\sigma_{e}^{4}}{12}}{\left(\frac{f_{e}^{2}}{6}\right)^{2}}+\frac{\left(\frac{\sigma_{e}^{2}}{2}\right)^{2}}{\left(\frac{\sigma_{e}^{2}}{6}\right)^{4}}\left(\frac{\sigma_{e}^{4}}{45}\right)\right)
$$

It simplifies to

$$
\sqrt{n} T\left(\not-1-\frac{3}{T}\right) \stackrel{d}{\rightarrow} N\left(0, \frac{51}{5}\right)
$$

as $(n, T) \rightarrow \infty$.

\section{E Proof of Theorem 5}

Proof. Let $S=\{T(1-\hat{\rho})>3\}$ and $\bar{S}=\{T(1-\hat{\rho}) \leq 3\}$. Consider (1). When $|\rho|<1$, it suffices to show that $\sqrt{n T}(\tilde{\rho}-\rho)-\sqrt{n T}\left(\not \phi-\rho+\frac{1+\hat{\rho}}{T}\right)=\sqrt{n T}\left(\not \phi-\hat{\rho}-\frac{1+\hat{\rho}}{T}\right) \stackrel{p}{\rightarrow} 0$. We have

$$
\begin{aligned}
\lim _{(n, T) \rightarrow \infty} \operatorname{Pr}\left(\left|\sqrt{n T}\left(\tilde{\rho}-\hat{\rho}-\frac{1+\hat{\rho}}{T}\right)\right|>\epsilon\right)= & \lim _{(n, T) \rightarrow \infty} \operatorname{Pr}\left(\left|\sqrt{n T}\left(\tilde{\rho}-\hat{\rho}-\frac{1+\hat{\rho}}{T}\right)\right|>\epsilon \mid S\right) \operatorname{Pr}(S) \\
& +\lim _{(n, T) \rightarrow \infty} \operatorname{Pr}\left(\left|\sqrt{n T}\left(\tilde{\rho}-\hat{\rho}-\frac{1+\hat{\rho}}{T}\right)\right|>\epsilon \mid \bar{S}\right) \operatorname{Pr}(\bar{S}) .
\end{aligned}
$$

The first term is zero given that, if $S$ is true, we have $\tilde{\rho}=\hat{\rho}+\frac{1+\hat{\rho}}{T}$ so that $\operatorname{Pr}\left(\left|\sqrt{n T}\left(\tilde{\rho}-\widehat{\rho}-\frac{1+\hat{\rho}}{T}\right)\right|>\epsilon \mid S\right) \mid=$ 0 . The second term is zero since $\sqrt{n T}\left(\hat{\rho}-\rho+\frac{1+\rho}{T}\right)=O_{p}(1)$ implies $\left.T(1-\hat{\rho})-3=T(1-\rho)+\sqrt{\frac{T}{n}}\left[\sqrt{n T}\left(\hat{\rho}-\rho+\frac{1+\rho}{T}\right)\right]-3-(1+\rho)=T(1-\rho)+O_{p} \quad \sqrt{\frac{T}{n}}\right)+o_{p}(1) \rightarrow \infty$ and hence $\operatorname{Pr}(\bar{S}) \rightarrow 0$ as $T \rightarrow \infty$. Therefore, $\operatorname{Pr}\left(\left|\sqrt{n T}\left(\tilde{\rho}-\hat{\rho}-\frac{1+\hat{\rho}}{T}\right)\right|>\epsilon\right) \rightarrow 0$ as $(n, T) \rightarrow \infty$.

Consider (2). When $|\rho|<1$, we have

$$
\begin{aligned}
\lim _{(n, T) \rightarrow \infty} \operatorname{Pr}(|\sqrt{n} T(\tilde{\rho}-1)|>\epsilon)= & \lim _{(n, T) \rightarrow \infty} \operatorname{Pr}(|\sqrt{n} T(\tilde{\rho}-1)|>\epsilon \mid S) \operatorname{Pr}(S) \\
& +\lim _{(n, T) \rightarrow \infty} \operatorname{Pr}(|\sqrt{n} T(\tilde{\rho}-1)|>\epsilon \mid \bar{S}) \operatorname{Pr}(\bar{S}) .
\end{aligned}
$$

Now the fact that $\sqrt{n} T\left(\hat{q}-1+\frac{3}{T}\right) \in O_{p}(1)$ implies $\operatorname{Pr}(S) \rightarrow 0$ as $(n, T) \rightarrow \infty$, so that the first term is zero. For the second term, if $\bar{S}$ is true, $\tilde{\rho}=1$ so that $\operatorname{Pr}(|\sqrt{n T}(\tilde{\rho}-1)|>\epsilon \mid \bar{S})=0$. Thus, $\operatorname{Pr}(|\sqrt{n} T(\tilde{\rho}-1)|>\epsilon) \rightarrow 0$ as $(n, T) \rightarrow \infty$. 


\section{Lemma 3}

$$
\begin{aligned}
\sum_{i=1}^{n} \sum_{t=2}^{T} \hat{t}_{i, t-1}^{2}= & \sum_{i=1}^{n} \sum_{t=2}^{T}\left(v_{i, t-1}-\overline{\bar{v}}\right)^{2}+\left(\hat{\beta}_{O L S}-\beta\right)^{2} \sum_{i=1}^{n} \sum_{t=2}^{T}(t-1-\bar{t})^{2} \\
& -2\left(\hat{\beta}_{O L S}-\beta\right) \sum_{i=1}^{n} \sum_{t=2}^{T}(t-1-\bar{t})\left(v_{i, t-1}-\overline{\bar{v}}\right)
\end{aligned}
$$

and

$$
\begin{aligned}
\sum_{i=1}^{n} \sum_{t=2}^{T}\left(\hat{u}_{i t}-\rho \hat{u}_{i, t-1}\right) \hat{u}_{i, t-1}= & \sum_{i=1}^{n} \sum_{t=2}^{T}\left[\left(\phi_{i t}-(1-\rho) \overline{\bar{v}}\right)\left(y_{i, t-1}-\overline{\bar{v}}\right)\right] \\
& +\left(\hat{\beta}_{O L S}-\beta\right) \sum_{j=1}^{n} \sum_{t=2}^{T}\left[(t-1-\bar{t})\left(\phi_{i t}-(1-\rho) \overline{\bar{v}}\right)\right] \\
& \left.+\left(\hat{\beta}_{O L S}-\beta\right) \sum_{i=1}^{n} \sum_{t=2}^{T}[(1-\rho)(t-\bar{t})+\rho)\left(v_{i, t-1}-\overline{\bar{v}}\right)\right] \\
& +\left(\hat{\beta}_{O L S}-\beta\right)^{2} \sum_{i=1}^{n} \sum_{t=2}^{T}[(t-1-\bar{t})((1-\rho)(t-\bar{t})+\rho)] .
\end{aligned}
$$

When $\rho=1$, it reduces to

$$
\begin{aligned}
\sum_{i=1}^{n} \sum_{t=2}^{T} \Delta \hat{u}_{i t} \hat{u}_{i, t-1}= & \sum_{i=1}^{n} \sum_{t=2}^{T}\left[e_{i t}\left(\chi_{i, t-1}-\overline{\bar{v}}\right)\right]\left(t \hat{\beta}_{O L S}-\beta\right) \sum_{i=1}^{n} \sum_{t=2}^{T}(t-1-\bar{t}) e_{i t} \\
& +\left(\hat{\beta}_{O L S}-\beta\right) \sum_{i=1}^{n} \sum_{t=2}^{T}\left(v_{i, t-1}-\overline{\bar{v}}\right)\left(t\left(\hat{\beta}_{O L S}-\beta\right)^{2} \sum_{i=1}^{n} \sum_{t=2}^{T}(t-1-\bar{t}),\right.
\end{aligned}
$$

where $\Delta \hat{u}_{i t}=\hat{u}_{i t}-\hat{u}_{i, t-1}$.

Proof. Since $\hat{u}_{i t}=\left(y_{i t}-\overline{\bar{y}}\right) f \hat{\beta}_{O L S}(t-\bar{t})=\left(v_{i t}-\overline{\bar{v}}\right)-\left(\hat{\beta}_{O L S}-\beta\right)(t-\bar{t})$, where $\overline{\bar{y}}=\frac{1}{n T} \sum_{i=1}^{n} \sum_{t=1}^{T} y_{i t}$ and $\overline{\bar{v}}=\frac{1}{n T} \sum_{i}^{n}=_{1} \sum_{t=1}^{T} v_{i t}$ and hence

$$
\begin{aligned}
& \hat{u}_{i t}-\rho \hat{u}_{i, t-1}
\end{aligned}
$$

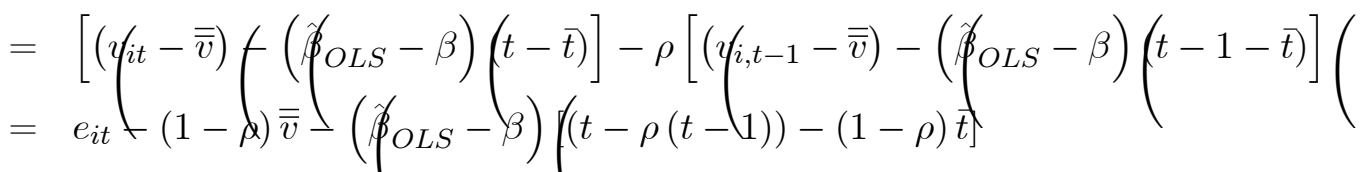

$$
\begin{aligned}
& =e_{i t}-(1-\rho) \overline{\bar{v}}-\left({ }_{O L S}-\beta\right)((1-\rho)(t-\bar{t})+\rho] \text {. }
\end{aligned}
$$

Results in Lemma 3 can be easily obtained.

Lemma 4 We have

$$
\sqrt{n} T^{3 / 2}\left(\ddot{\beta}_{O L S}-\beta\right) \stackrel{d}{\rightarrow} N\left(\phi, \frac{12 \sigma_{e}^{2}}{(1-\rho)^{2}}\right)(
$$


when $|\rho|<1$ and

$$
\sqrt{n T}\left(\hat{\beta}_{O L S}-\beta\right) \stackrel{d}{\rightarrow} N\left(0, \frac{6}{5} \sigma_{e}^{2}\right)
$$

when $\rho=1$.

Proof. Using $u_{i t}=\mu_{i}+v_{i t}$, we have

$$
\hat{\beta}_{O L S}-\beta=\frac{\sum_{i=1}^{n} \sum_{t=1}^{T}(t-\bar{t}) u_{i t}}{\sum_{i=1}^{n} \sum_{t=1}^{T}(t-\bar{t})^{2}}=\frac{\sum_{i=1}^{n} \sum_{t=1}^{T}(t-\bar{t}) v_{i t}}{\sum_{k=1}^{n} \sum_{t=1}^{T}(t-\bar{t})^{2}}
$$

since $\sum_{i=1}^{n} \sum_{t=1}^{T}(t-\bar{t}) \mu_{i}=\sum_{i=1}^{n} \mu_{i} \sum_{t=1}^{T}(t-\bar{t})=0$. By Theorems 3 and 4 in Kao and Emerson (2004), we have the asymptotic property of $\hat{\beta}_{O L S}$ in Lemma 4.

\section{F $\quad$ Proof of Theorem 6}

Proof. Consider (1). Note that

From Lemma 3, we have

$$
\hat{\rho}-\rho=\frac{\sum_{i}^{n}\left(\sum_{1} \sum_{t=2}^{T}\left(\hat{u}_{i t}-\rho \hat{u}_{i, t-1}\right) \hat{u}_{i, t-1}\right.}{\sum_{i=1}^{n} \sum_{t=2}^{T} \hat{u}_{i, t-1}^{2}} .
$$

$$
\begin{aligned}
\frac{1}{n T} \sum_{i=1}^{n} \sum_{t=2}^{T} \hat{u}_{i, t-1}^{2}= & \frac{1}{n T} \sum_{i=1}^{n} \sum_{t=2}^{T}\left(v_{i, t-1}-\overline{\bar{v}}\right)^{2}+\frac{1}{n T}\left[\sqrt{n} T^{3 / 2}\left(\hat{\beta}_{O L S}-\beta\right)\right]^{2}\left[\frac{1}{n T^{3}} \sum_{i=1}^{n} \sum_{t=2}^{T}(t-1-\bar{t})^{2}\right]( \\
& -\frac{2}{n T}\left[\sqrt{n} T^{3 / 2}\left(\hat{\beta}_{O L S}-\beta\right)\right]\left(\frac{1}{\sqrt{n} T^{3 / 2}} \sum_{i=1}^{n} \sum_{t=2}^{T}(t-1-\bar{t})\left(v_{i t}-\overline{\bar{v}}\right)\right](
\end{aligned}
$$

Notice that

$$
\frac{1}{n T} \sum_{i=1}^{n} \sum_{t=2}^{T}\left(y_{i, t-1}-\overline{\bar{v}}\right)^{2} \stackrel{p}{\rightarrow} \frac{\sigma_{e}^{2}}{1-\rho^{2}}
$$

from Lemma 3.1 of Baltagi, Kao and Liu (2008),

$$
\begin{gathered}
\frac{1}{n T^{3}} \sum_{i=1}^{n} \sum_{t=2}^{T}(t-1-\bar{t})^{2} \stackrel{p}{\rightarrow} \frac{1}{12}, \\
\frac{1}{\sqrt{n} T^{3 / 2}} \sum_{i=1}^{n} \sum_{t=2}^{T}(t-1-\bar{t})\left(v_{i t}-\overline{\bar{v}}\right)\left(=\frac{1}{\sqrt{n} T^{3 / 2}} \sum_{i=1}^{n} \sum_{t=2}^{T}(t-1-\bar{t}) v_{i t}+o_{p}(1) \stackrel{d}{\rightarrow} \frac{\sigma_{e}^{2}}{1-\rho^{2}} N\left(0, \frac{1}{12}\right),\right.
\end{gathered}
$$

and

$$
\sqrt{n} T^{3 / 2}\left(\hat{\beta}_{O L S}-\beta\right) \stackrel{d}{\rightarrow} N\left(0, \frac{12 \sigma_{e}^{2}}{(1-\rho)^{2}}\right)
$$

by Lemma 4 . 
Hence we have

$$
\frac{1}{n T} \sum_{i=1}^{n} \sum_{t=2}^{T} \hat{\iota}_{i, t-1}^{2} \stackrel{p}{\rightarrow} \frac{\sigma_{e}^{2}}{1-\rho^{2}}
$$

as $(n, T) \rightarrow \infty$. Also, from Lemma 3 , we have

$$
\begin{aligned}
& \frac{1}{\sqrt{n T}} \sum_{i=1}^{n} \sum_{t=2}^{T}\left(\hat{u}_{i t}-\rho \hat{u}_{i, t-1}\right) \hat{u}_{i, t-1} \\
= & \frac{1}{\sqrt{n T}} \sum_{i=1}^{n} \sum_{t=2}^{T}\left[\left(\phi_{i t}-(1-\rho) \overline{\bar{v}}\right)\left(y_{i, t-1}-\overline{\bar{v}}\right)\right] \\
& +\frac{1}{\sqrt{n T}}\left[\sqrt{n} T^{3 / 2}\left(\tilde{\phi}_{O L S}-\beta\right)\right]\left(\frac{1}{\sqrt{n} T^{3 / 2}} \sum_{i=1}^{n} \sum_{t=2}^{T}\left[(t-1-\bar{t})\left(\phi_{i t}-(1-\rho) \overline{\bar{v}}\right)\right]\right. \\
& +\frac{1}{\sqrt{n T}}\left[\sqrt{n} T^{3 / 2}\left(\tilde{\phi}_{O L S}-\beta\right)\right]\left(\frac{1}{\sqrt{n} T^{3 / 2}} \sum_{i=1}^{n} \sum_{t=2}^{T}[(1-\rho)(t-\bar{t})+\rho)\left(v_{i, t-1}-\overline{\bar{v}}\right)\right] \\
& +\frac{1}{\sqrt{n T}}\left[\sqrt{n} T^{3 / 2}\left(\tilde{\phi}_{O L S}-\beta\right)\right]^{2} \frac{1}{n T^{3}} \sum_{i=1}^{n} \sum_{t=2}^{T}[(t-1-\bar{t})((1-\rho)(t-\bar{t})+\rho)] .
\end{aligned}
$$

Notice that

$$
\begin{aligned}
& \frac{1}{\sqrt{n T}} \sum_{i=1}^{n} \sum_{t=2}^{T}\left[\left(\phi_{i t}-(1-\rho) \overline{\bar{v}}\right)\left(y_{i, t-1}-\overline{\bar{v}}\right)\right] \\
&= \frac{1}{\sqrt{n T}} \sum_{i=1}^{n} \sum_{t=2}^{T} \oint_{i t} v_{i, t-1}-\frac{1}{\sqrt{n T}}(\sqrt{n T} \overline{\bar{v}})\left[\frac{1}{\sqrt{n T}} \sum_{i=1}^{n} \sum_{t=2}^{T} \oint_{i t}+(1-\rho) \frac{1}{\sqrt{n T}} \sum_{i=1}^{n} \sum_{t=2}^{T} v_{i, t-1}\right]( \\
&+\frac{1}{\sqrt{n T}}(1-\rho)(\sqrt{n T} \overline{\bar{v}})^{2} \\
& \stackrel{d}{\rightarrow} \sigma_{e}^{2} N\left(0, \frac{1}{1-\rho^{2}}\right)(+o(1), \\
& \frac{1}{\sqrt{n} T^{3 / 2}} \sum_{i=1}^{n} \sum_{t=2}^{T}\left[(t-1-\bar{t})\left(q_{i t}-(1-\rho) \overline{\bar{v}}\right)\right]\left(=\frac{1}{\sqrt{n} T^{3 / 2}} \sum_{i=1}^{n} \sum_{t=2}^{T}\left[(t-1-\bar{t}) e_{i t}\right]+o_{p}(1) \stackrel{d}{\rightarrow} \sigma_{e}^{2} N\left(0, \frac{1}{12}\right),\right. \\
& \frac{1}{n T^{3}} \sum_{i=1}^{n} \sum_{t=2}^{T}[(t-1-\bar{t})((1-\rho)(t-\bar{t})+\rho)]=(1-\rho) \frac{1}{n T^{3}} \sum_{i=1}^{n} \sum_{t=2}^{T}(t-\bar{t})^{2}+o_{p}(1) \stackrel{p}{\rightarrow} \frac{1-\rho}{12},
\end{aligned}
$$

and

$$
\sqrt{n} T^{3 / 2}\left(\hat{\beta}_{O L S}-\beta\right) \stackrel{d}{\rightarrow} N\left(0, \frac{12 \sigma_{e}^{2}}{(1-\rho)^{2}}\right)
$$

by Lemma 4 .

Hence we have

$$
\frac{1}{\sqrt{n T}} \sum_{i=1}^{n} \sum_{t=2}^{T}\left(\hat{u}_{i t}-\rho \hat{u}_{i, t-1}\right) \hat{u}_{i, t-1} \stackrel{d}{\rightarrow} \sigma_{e}^{2} N\left(0, \frac{1}{1-\rho^{2}}\right)
$$


as $(n, T) \rightarrow \infty$. Therefore,

as $(n, T) \rightarrow \infty$.

$$
\sqrt{n T}(\hat{\rho}-\rho)=\frac{\frac{1}{\sqrt{n T}} \sum_{i}^{n}\left(\sum_{1} \sum_{t=2}^{T}\left(\hat{u}_{i t}-\rho \hat{u}_{i, t-1}\right) \hat{u}_{i, t-1}\right.}{\frac{1}{\alpha T} \sum_{i}^{n}\left({ }_{1} \sum_{t=2}^{T} \hat{u}_{i, t-1}^{2}\right.} \stackrel{d}{\rightarrow} \frac{\sigma_{e}^{2} N\left(0, \frac{1}{1-\rho^{2}}\right)}{\frac{\sigma_{e}^{2}}{1-\rho^{2}}}=N\left(0,1-\rho^{2}\right)
$$

Consider (2). Note that

$$
\hat{\rho}-1=\frac{\sum_{i=1}^{n} \sum_{t=2}^{T} \Delta \hat{u}_{i t} \hat{u}_{i, t-1}}{\sum_{i=1}^{n} \sum_{t=2}^{T} \hat{u}_{i, t-1}^{2}} .
$$

From Lemma 3, we have

$$
\begin{aligned}
\frac{1}{n T^{2}} \sum_{i=1}^{n} \sum_{t=2}^{T} \hat{\mu}_{i, t-1}^{2}= & \frac{1}{n T^{2}} \sum_{i=1}^{n} \sum_{t=2}^{T}\left(y_{i, t-1}-\overline{\bar{v}}\right)^{2}+\frac{1}{n}\left[n T\left(\tilde{\beta}_{O L S}-\beta\right)\right]^{2}\left[\frac{1}{n T^{3}} \sum_{i=1}^{n} \sum_{t=2}^{T}(t-1-\bar{t})^{2}\right]( \\
& -\frac{2}{n}\left[\sqrt{n T}\left(\hat{\beta}_{O L S}-\beta\right)\right]\left[\frac{1}{\sqrt{n} T^{5 / 2}} \sum_{i=1}^{n} \sum_{t=2}^{T}(t-1-\bar{t})\left(v_{i, t-1}-\overline{\bar{v}}\right)\right](
\end{aligned}
$$

Notice that

by equation (C3) in Kao (1999),

$$
\frac{1}{n T^{2}} \sum_{i=1}^{n} \sum_{t=2}^{T}\left(y_{i, t-1}-\overline{\bar{v}}\right)^{2} \stackrel{p}{\rightarrow} \frac{\sigma_{e}^{2}}{2}
$$

$$
\sqrt{n T}\left(\hat{\beta}_{O L S}-\beta\right) \stackrel{d}{\rightarrow} N\left(0, \frac{6}{5} \sigma_{e}^{2}\right)
$$

by Lemma 4 ,

$$
\frac{1}{\sqrt{n} T^{5 / 2}} \sum_{i=1}^{n} \sum_{t=2}^{T}(t-1-\bar{t})\left(v_{i, t-1}-\overline{\bar{v}}\right)\left(=\frac{1}{\sqrt{n} T^{5 / 2}} \sum_{i=1}^{n} \sum_{t=2}^{T}\left[(t-1-\bar{t}) v_{i, t-1}\right]+o_{p}(1) \stackrel{d}{\rightarrow} N\left(0, \frac{\sigma_{e}^{2}}{120}\right)\right.
$$

by an equation on page 23 in Kao and Emerson (2004) and

$$
\frac{1}{n T^{3}} \sum_{i=1}^{n} \sum_{t=2}^{T}(t-1-\bar{t})^{2} \rightarrow \frac{1}{12}
$$

Hence we have

$$
\begin{aligned}
\frac{1}{n T^{2}} \sum_{i=1}^{n} \sum_{t=2}^{T} \hat{f}_{i, t-1}^{2}= & \frac{1}{n T^{2}} \sum_{i=1}^{n} \sum_{t=2}^{T}\left(y_{i, t-1}-\overline{\bar{v}}\right)^{2}+\frac{1}{n}\left[n T\left(\hat{\beta}_{O L S}-\beta\right)\right]^{2}\left[\frac{1}{n T^{3}} \sum_{i=1}^{n} \sum_{t=2}^{T}(t-1-\bar{t})^{2}\right]( \\
& -\frac{2}{n}\left[\sqrt{n T}\left(\hat{\beta}_{O L S}-\beta\right)\right]\left[\frac{1}{\sqrt{n} T^{5 / 2}} \sum_{i=1}^{n} \sum_{t=2}^{T}(t-1-\bar{t})\left(v_{i, t-1}-\overline{\bar{v}}\right)\right]\left(\left(\stackrel{p}{\rightarrow} \frac{\sigma_{e}^{2}}{6}\right.\right.
\end{aligned}
$$


as $(n, T) \rightarrow \infty$. Also, from Lemma 3 ,

$$
\begin{aligned}
& \frac{1}{\sqrt{n} T} \sum_{i=1}^{n} \sum_{t=2}^{T} \Delta \hat{u}_{i t} \hat{u}_{i, t-1} \\
= & \frac{1}{\sqrt{n} T} \sum_{i=1}^{n} \sum_{t=2}^{T}\left[e_{i t}\left(\chi_{i, t-1}-\overline{\bar{v}}\right)\right]\left(\frac{1}{\sqrt{n}}\left[\sqrt{n T}\left(\hat{\beta}_{O L S}-\beta\right)\right]\left[\frac{1}{\sqrt{n} T^{3 / 2}} \sum_{i=1}^{n} \sum_{t=2}^{T}(t-1-\bar{t}) e_{i t}\right](\right. \\
+ & \frac{1}{T^{3 / 2}}\left[\sqrt{n T}\left(\ddot{\phi}_{O L S}-\beta\right)\right]\left[\frac{1}{\sqrt{n} T^{3 / 2}} \sum_{i=1}^{n} \sum_{t=2}^{T}\left(\chi_{i, t-1}-\overline{\bar{v}}\right)\right]( \\
+ & \frac{1}{\sqrt{n} T}\left[\sqrt{n T}\left(\hat{\phi}_{O L S}-\beta\right)\right]^{2}\left[\frac{1}{n T} \sum_{i=1}^{n} \sum_{t=2}^{T}(t-1-\bar{t})\right](
\end{aligned}
$$

Notice that

$$
\begin{aligned}
& \frac{1}{\sqrt{n} T} \sum_{i=1}^{n} \sum_{t=2}^{T}\left[e_{i t}\left(\chi_{i, t-1}-\overline{\bar{v}}\right)\right] \\
& \left.\left.=\frac{1}{\sqrt{n} T} \sum_{i=1}^{n} \sum_{t=2}^{T} \xi_{i t} v_{i t}-\frac{1}{\sqrt{n}} \frac{1}{\sqrt{n T}} \sum_{i=1}^{n} \sum_{t=2}^{T} e_{i, t-1}\right) \quad \frac{1}{\sqrt{n} T^{3 / 2}} \sum_{i=1}^{n} \sum_{t=2}^{T} v_{i t}\right)( \\
& \stackrel{d}{\rightarrow} N\left(0, \frac{\sigma_{e}^{2}}{12}\right)( \\
& \frac{1}{\sqrt{n} T^{3 / 2}} \sum_{i=1}^{n} \sum_{t=2}^{T}(t-1-\bar{t}) e_{i t} \stackrel{d}{\rightarrow} N\left(0, \frac{\sigma_{e}^{2}}{12}\right) \\
& \frac{1}{\sqrt{n} T^{3 / 2}} \sum_{i=1}^{n} \sum_{t=2}^{T}\left(y_{i, t-1}-\overline{\bar{v}}\right) \stackrel{d}{\rightarrow} N\left(\oint, \frac{\sigma_{e}^{2}}{3}\right)
\end{aligned}
$$

and

$$
\sqrt{n T}\left(\hat{\beta}_{O L S}-\beta\right) \stackrel{d}{\rightarrow} N\left(0, \frac{6}{5} \sigma_{e}^{2}\right)
$$

by Lemma 4 and

$$
\frac{1}{n T} \sum_{i=1}^{n} \sum_{t=2}^{T}(t-1-\bar{t}) \stackrel{p}{\rightarrow} \frac{1}{2}
$$

Hence we have

$$
\frac{1}{\sqrt{n} T} \sum_{i=1}^{n} \sum_{t=2}^{T} \widehat{\triangle} \hat{u}_{i t} \hat{u}_{i, t-1} \stackrel{d}{\rightarrow} N\left(0, \frac{\sigma_{e}^{2}}{12}\right)
$$

as $(n, T) \rightarrow \infty$. Therefore,

as $(n, T) \rightarrow \infty$.

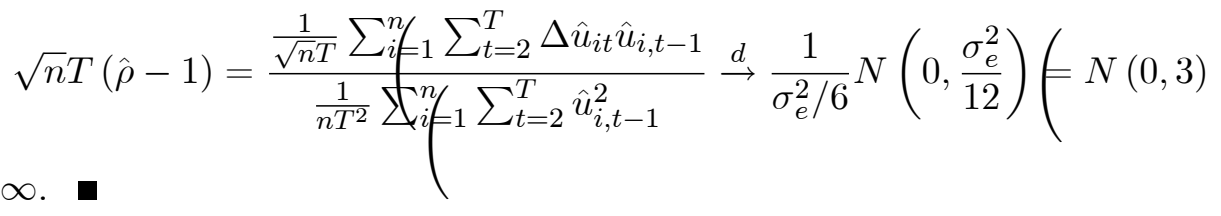

